ARTICLE

https://doi.org/10.1038/s41467-019-09940-1

\title{
Functional linkage of gene fusions to cancer cell fitness assessed by pharmacological and CRISPR- Cas9 screening
}

\author{
Gabriele Picco 1,9, Elisabeth D. Chen (10) 1,9, Luz Garcia Alonso (10 2,3, Fiona M. Behan (1) 1,3, Emanuel Gonçalves ${ }^{1}$, \\ Graham Bignell', Angela Matchan¹, Beiyuan Fu¹, Ruby Banerjee ${ }^{1}$, Elizabeth Anderson ${ }^{1}$, Adam Butler ${ }^{1}$, \\ Cyril H. Benes ${ }^{4}$, Ultan McDermott1,5, David Dow 3,6,7, Francesco lorio (1) 1,2,3, Euan Stronach 3,6,7, \\ Fengtang Yang (1) ${ }^{1}$, Kosuke Yusa ${ }^{1}$, Julio Saez-Rodriguez (D) ${ }^{2,3,8}$ \& Mathew J. Garnett ${ }^{1,3}$
}

\begin{abstract}
Many gene fusions are reported in tumours and for most their role remains unknown. As fusions are used for diagnostic and prognostic purposes, and are targets for treatment, it is crucial to assess their function in cancer. To systematically investigate the role of fusions in tumour cell fitness, we utilized RNA-sequencing data from 1011 human cancer cell lines to functionally link 8354 fusion events with genomic data, sensitivity to $>350$ anti-cancer drugs and CRISPR-Cas9 loss-of-fitness effects. Established clinically-relevant fusions were identified. Overall, detection of functional fusions was rare, including those involving cancer driver genes, suggesting that many fusions are dispensable for tumour fitness. Therapeutically actionable fusions involving RAF1, BRD4 and ROS1 were verified in new histologies. In addition, recurrent YAP1-MAML2 fusions were identified as activators of Hippo-pathway signaling in multiple cancer types. Our approach discriminates functional fusions, identifying new drivers of carcinogenesis and fusions that could have clinical implications.
\end{abstract}

\footnotetext{
${ }^{1}$ Wellcome Sanger Institute, Wellcome Genome Campus, Cambridge CB10 1SA, UK. ${ }^{2}$ European Molecular Biology Laboratory, European Bioinformatics Institute, Wellcome Genome Campus, Cambridge CB10 1SD, UK. ${ }^{3}$ Open Targets, Wellcome Genome Campus, Cambridge CB10 1SA, UK. ${ }^{4}$ Massachusetts General Hospital, 55 Fruit Street, Boston, MA 02114, USA. ${ }^{5}$ AstraZeneca, CRUK Cambridge Institute, Cambridge CB2 ORE, UK. ${ }^{6}$ Research and Development, GlaxoSmithKline, Stevenage SG1 2NY, UK. ${ }^{7}$ Research and Development, GlaxoSmithKline, Collegeville, PA 19426-0989, USA. ${ }^{8}$ Institute for Computational Biomedicine, Faculty of Medicine, Bioquant, Heidelberg University, 69120 Heidelberg, Germany. ${ }^{9}$ These authors contributed equally: Gabriele Picco, Elisabeth D. Chen. Correspondence and requests for materials should be addressed to M.J.G. (email: mathew.garnett@sanger.ac.uk)
} 
O ncogenic gene fusions occur in solid tumours and hematologic malignancies, and are used for diagnostic purposes, patient risk stratification, and for monitoring of residual disease ${ }^{1}$. Critically, the chimeric protein encoded by fusions may be a tumour-specific target for treatment, resulting in significant clinical benefit for patients ${ }^{2,3}$. Fusions are often associated with a tissue histology, but can occur at a low frequency in multiple histologies. Gene fusion transcripts are composed of two independent genes formed either through structural rearrangements, transcriptional read-through of adjacent genes, or premessenger RNA (mRNA) splicing. The exchange of coding or regulatory sequences between genes can result in aberrant functionality of the fusion protein, and deregulation of the partner genes, including overexpression of oncogenes and decreased expression of tumour suppressor genes (TSGs).

Discriminating between fusions that have a role in cancer fitness and those that do not is a major challenge with important clinical implications ${ }^{4}$. Deep sequencing technology together with sensitive fusion detection algorithms have led to a dramatic increase in the number of reported cancer-associated fusions ${ }^{5}$. Most fusion transcripts are likely the indirect consequence of genomic instability or false-positive events due to error-prone fusion calling. Previous studies have focused on the identification of fusions, or have investigated the function of specific gene fusions; for example, in the setting of acute myeloid leukemia $(\mathrm{AML})^{6}$. The functional role of most cancer-associated fusions has not been investigated.

We have generated large-scale genomic and pharmacological datasets for over 1000 human cancer cell lines as part of the Genomics of Drug Sensitivity in Cancer (GDSC) project ${ }^{7,8}$. These datasets, together with CRISPR-Cas9 genetic screening technology, make it now possible to systematically assess the contribution of fusion transcripts to cancer cell fitness. Here, we report a comprehensive functional landscape of fusions using RNAsequencing (RNA-seq) data for 1011 human cancer cell lines. We investigate the functional relevance of gene fusions using differential gene expression, drug sensitivity to $>350$ anti-cancer compounds, and whole-genome CRISPR-Cas9 drop-out screens to identify fusions required for cancer cell fitness. To our knowledge, this study is the first large-scale systematic analysis in human cancer models to unveil the largely unexplored functional role of gene fusions.

\section{Results}

Landscape of fusion transcripts. To systematically identify gene fusions in diverse cancer types, we first analyzed RNA-seq data to define fusion transcripts in the GDSC cancer cell lines (1034 samples from 1011 unique cell lines) representing 41 cancer types (Fig. 1 and Supplementary Data 1$)^{9}$. RNA-seq data for 587 cell lines was obtained from the Cancer Genome Hub (CGHub) and 447 cell lines were sequenced at the Sanger Institute ${ }^{10}$. Fusion calling algorithms are prone to detecting false positives from sequencing artifacts and alignment ambiguities ${ }^{11}$. To improve the accuracy of fusion transcript calling, we used three different algorithms, deFuse, TopHat-Fusion, and STAR-Fusion, across all samples ${ }^{12-14}$ and applied stringent filtering criteria. In total, 10,514 fusion transcripts were called by more than one algorithm and taken forward for this study (Fig. $1 \mathrm{~b}$ and Supplementary Data 2). Targeted PCR of 406 putative fusion breakpoints indicated a validation rate of $72 \%$. Furthermore, we compared 23 samples with RNA-seq data from both Sanger Institute and CGHub (Supplementary Data 1), and the proportion of fusions transcripts in both data sources for a given cell line was $70 \%$. The presence of a fusion in a cell line, even in instances where multiple transcripts involving the same partner genes were detected, was defined as a "fusion event". Thus, we identified 8354 gene fusion events from 10,514 fusion transcripts and, because only a small number of fusions were recurrent, a total of 7430 unique fusions (Supplementary Data 2).

Next, we examined the number of fusion events that occurred in different cancer types. Cell lines had a median of six fusion events and $26 \%$ of fusion events were predicted to be in frame. Fusion numbers varied by cancer type (Fig. 1c), with osteosarcoma and breast cancer having the most (median of 16 fusion events per cell line), and kidney cancers and B-lymphoblastic leukemia together with three non-cancerous immortalized human cell lines having the lowest number of fusion events (median $=2$ ). The prevalence of fusion events for each cancer type in our cell lines was slightly higher, but significantly correlated with the frequency reported from the analysis of 9624 patient samples $(p<$ $0.001, R^{2}=0.42$, Pearson's correlation; Supplementary Fig. 1a), indicating that cell lines reflect the frequency of fusions in tumours from different tissues ${ }^{15}$. We identified recurrent known oncogenic fusions events, including $B C R-A B L 1$ ( $n=11$ cell lines), NPM1-ALK $(n=5)$, EWSR1-FLI1 $(n=24)$, and TMPRSS2-ERG $(n=2)$. Of note, only 431 of $7430(6 \%)$ fusions were recurrent, while the remaining were detected in only one cell line (Fig. 1d), indicating that most fusions are rare.

Of the fusion events we identified, $11 \%$ have been reported in human tumour samples ${ }^{15}$. For $14.2 \%$ of the fusion events, at least one of the fused genes is in the COSMIC Cancer Gene Census, representing an enrichment for cancer genes (odds ratio $=1.8$ and $p<0.001$, Fisher's test). TSGs were enriched as $5^{\prime}$ end partner genes (odds ratio $=2.1$ and $p<0.001$, Fisher's test), while oncogenes were enriched as $3^{\prime}$ or $5^{\prime}$ genes (odds ratio $=2$ and 1.8 , respectively, $p<0.001$, Fisher's test). An empirical permutation test identified known oncogenic fusions enriched in specific cancer types that are consistent with their pathognomonic nature, such as $A B L 1$ fusions in chronic myeloid leukemia (false discovery rate (FDR) $<1 \%, n=9$ ), EWSR1-FLI1 fusions in Ewing's sarcoma (FDR $<1 \%, n=24$ ) and FGFR3 fusions in bladder cancer (FDR $<1 \%, n=3$ ) (Supplementary Fig. 1b). In summary, using multiple algorithms and stringent criteria we built a comprehensive landscape of fusions in cancer cell lines, most of which occur at a low frequency, and reflect the prevalence and tissue specificity in tumour samples.

Fusions impact gene expression. Fusions may result in altered expression of fusion partner genes ${ }^{15}$. To identify genes whose expression is altered when fused, we first aggregated fusion events that had a common gene partner at the $5^{\prime}$ or the $3^{\prime}$ end to increase sample size and statistical power. We then used multiple linear regression (MLR) to link gene expression with the presence of a fused gene, incorporating bias due to copy number alterations and cancer type. In total, we tested 902 genes $\left(5^{\prime}\right.$ genes $n=$ 611 and $3^{\prime}$ genes $n=383$ ) that involved 3048 fusions. We identified $172(19 \%)$ genes significantly associated with differential expression $\left(5^{\prime}\right.$ genes $=54(9 \%)$ and $3^{\prime}$ genes $\left.n=118(31 \%)\right)$ that encompassed 592 fusions (Fig. 2a). Of the significantly associated genes, $24(14 \%)$ were from the COSMIC cancer gene census (2.5\% of the total; Fig. 2a and Supplementary Data 3). As expected, several TSGs such as TP53, APC, and KDM6A were significantly associated with reduced expression $(p<0.001$, MLR, Supplementary Fig. 1c). In contrast, many oncogenes fused at the $3^{\prime}$ end were overexpressed, including $A L K, E R G, F L 1, M Y C$, $M L L 4$, and ROS1 ( $p<0.001$, MLR, Supplementary Fig. 1c).

Because most fusions are rare and therefore not suitable for linear regression modeling, we also annotated expression of genes involved in each fusion event $(n=8354)$. We focused on $3^{\prime}$ end genes with exceptionally high expression because overexpression 
a

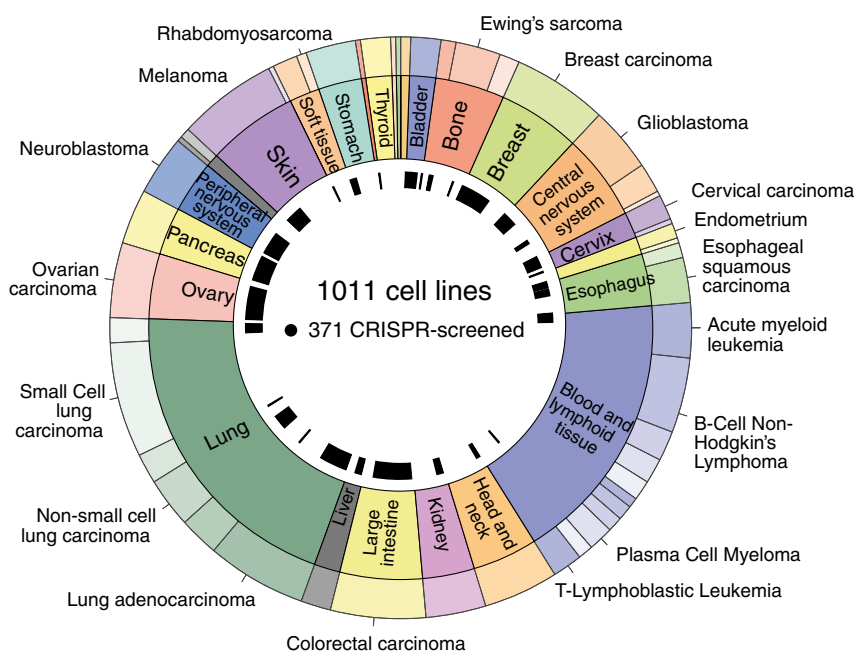

。

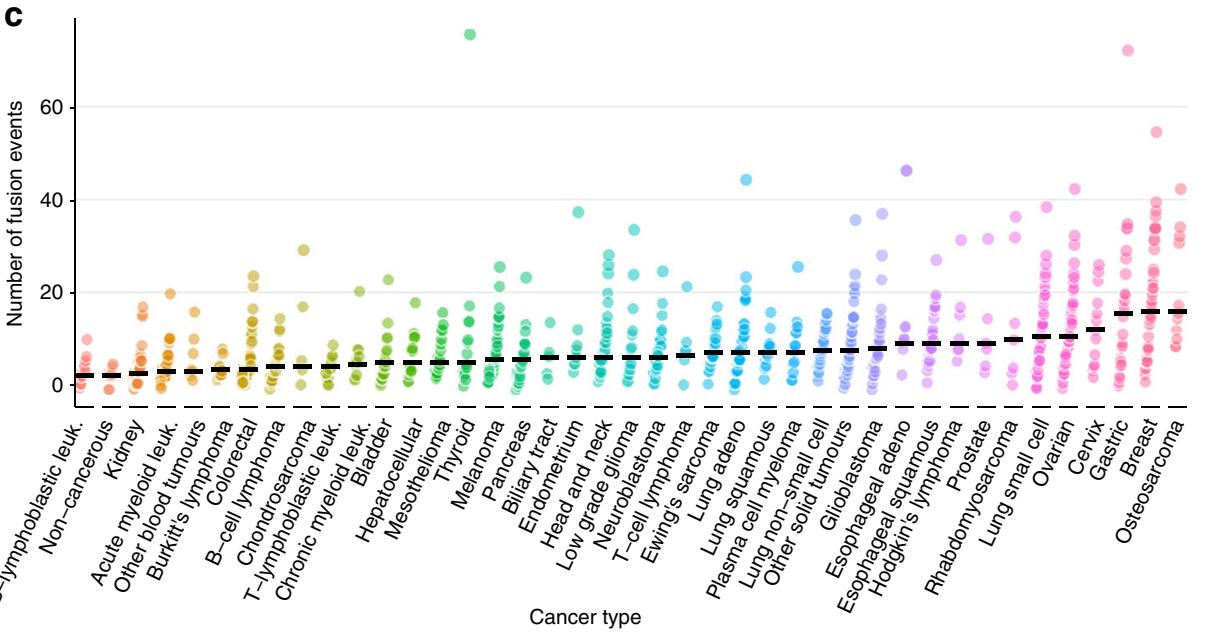

b

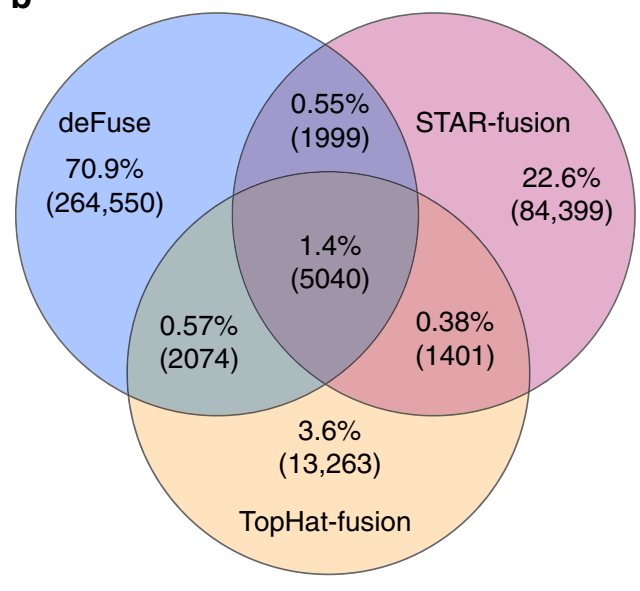

d

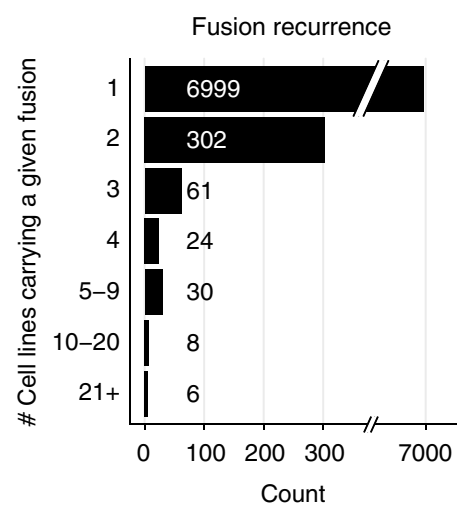

Fig. 1 Landscape of gene fusions in cancer cell lines. a Tissues (inner ring) and cancer types (outer ring) represented by the cell lines and CRISPR dataset used for this study. b Fusion transcript calls using three algorithms and their overlap. c Frequency of gene fusion events in cancer cell lines, separated by cancer type. The black line is the median. d Fusion event recurrence in cancer cell lines

a

Fusion events with a common gene partner at the $5^{\prime}$ or the $3^{\prime}$ end $5^{\prime}$ or $3^{\prime}$ differentially expressed

Driver gene

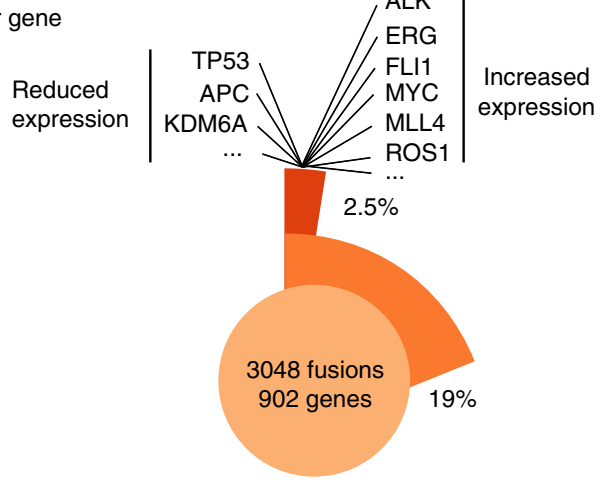

b

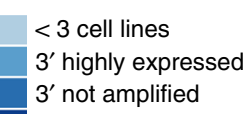

3' not amplified

$3^{\prime}$ is a driver gene
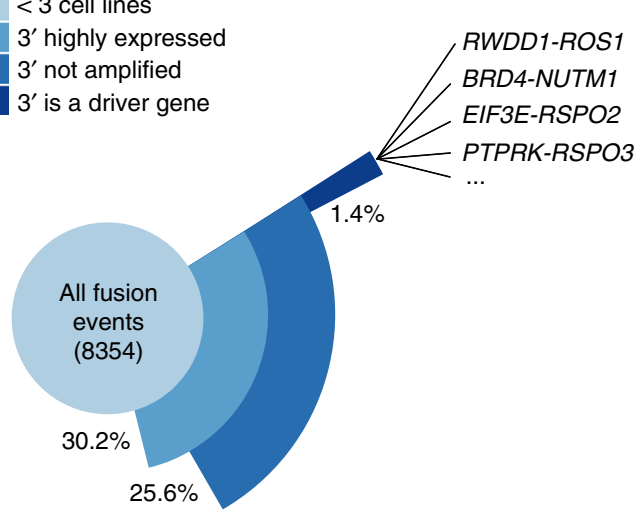

Fig. 2 Fusions impact gene expression. a Frequency of a statistical association between a recurrent fusion ( $n>2$ cell lines) and differential gene expression. Examples of downregulated tumour suppressor genes (TSGs) and overexpressed oncogenes are displayed. b Frequency of co-occurrence of a gene fusion and overexpression of the 3' gene for each fusion event. RSPO2, RSPO3, and NUTM1 are examples of overexpressed cancer driver genes involved in previously unreported gene fusions 
of proto-oncogenes occurring as $3^{\prime}$ partner genes is observed in several malignancies ${ }^{15,16}$. We found that $25.6 \%(n=2145)$ of fusion events were coincident with high expression and did not co-occur with copy number amplification (Fig. 2b). Only $5.4 \%$ (1.4\% of the total; $n=117$ ) of these fusion events involve the overexpression of a COSMIC driver gene (Fig. $2 b$ and Supplementary Data 4). Thus, aberrant transcript expression of genes involved in gene fusions is a common event, but only a small subset of these fusions involve established driver oncogenes.

This analysis identified new contexts for fusion transcripts leading to overexpression of cancer genes located at the fusion $3^{\prime}$ end, such as NUTM1, RSPO2/3, and ROS1 (Fig. 2b). In support of this observation, we validated by Sanger sequencing and fluorescence in situ hybridization (FISH) a previously uncharacterized RWDD1-ROS1 fusion in the OCUB-M cell line, which is derived from a triple-negative breast cancer (Supplementary Fig. 2a and b). ROS1 is a receptor tyrosine kinase and gene rearrangements leading to ROS1 overexpression are therapeutic biomarkers of response to ROS1 kinase inhibitors in non-smallcell lung cancer and other cancer types (Supplementary Fig. $2 \mathrm{c})^{17}$. The fusion retains the ROS1 protein kinase domain and OCUB$M$ cells display sensitivity to crizotinib and foretinib, two potent ROS1 inhibitors (Supplementary Fig. 2d and e) ${ }^{18,19}$. Interestingly, in a dataset of 590 breast cancer patients, we identified a triplenegative and a HER2+ tumour-carrying in-frame fusions involving the ROS1 kinase domain ${ }^{20}$ (Supplementary Fig. 2f), suggesting that this rare subset of breast cancer patients could be potentially eligible to targeted tyrosine kinase inhibitor-based therapies.

Fusions as markers of drug sensitivity. Fusion proteins can impact on clinical responses to therapy. Consequently, we reasoned that differential drug sensitivity in cell lines could be used to identify functional fusions, as well as opportunities for repurposing of existing drugs. We used an established statistical model $^{8,21}$ to perform an analysis of variance (ANOVA) linking the 431 recurrent gene fusions ( $n \geq 2$ cell lines; in-frame and not in frame fusions) with $308,634 \mathrm{IC}_{50}$ (half-maximal inhibitory concentration) values for 409 anti-cancer drugs (334 unique compounds) screened across 982 cell lines by the GDSC project (Fig. 3a and Supplementary Data 5). The compounds assessed consisted of anti-cancer chemotherapeutics and molecularly targeted agents, including many which are Food and Drug Administration-approved $(n=46)$ or in clinical development ( $n=65$; Fig. 3a). This included data for 155 new compounds and a total of 212,774 previously unpublished $\mathrm{IC}_{50}$ values. Preliminary analyses indicated that mutations in cancer driver genes cooccurring with fusions in cell lines were frequent confounders when identifying fusion-specific associations. To control for this, we first identified associations between 717 cancer driver mutations and copy number alterations with drug sensitivity, and then used them as a covariate in the ANOVA to identify fusionspecific associations (Supplementary Data 6). Adding the covariates resulted in 11 fusion associations falling below our threshold for statistical significance. For instance, the association of NKD1-ADCY7 with BRAF-inhibitor dabrafenib was explained by the presence of a coincident $B R A F$ mutation in one highly sensitive cell line (Supplementary Fig. 3a).

We identified 227 large-effect size associations (ANOVA FDR $<25 \%$ and Glass Delta's $>1$; the Glass Delta's are a measure of effect size incorporating the standard deviation of the two subpopulations) between gene fusions and drug sensitivity (Fig. 3b; Supplementary Data 7). At the level of individual fusion events, 284 of $1355(21 \%)$ tested fusion events were involved in a significant association with a drug. Most of the strongest fusion-drug associations were well understood cases, such as sensitivity of $A L K$-fusion-positive cell lines to ALK inhibitors, for example, alectinib, (FDR $<0.1 \%$ ), and sensitivity of $B C R-A B L 1$ translocation-positive cells to $\mathrm{ABL}$ inhibitors, such as imatinib and nilotinib (FDR <0.1\%) (Fig. 3b, c). We also identified associations with low-frequency fusions, such as sensitivity to multiple EGFR inhibitors (e.g., cetuximab), in two CRTC1MAML2-fusion-positive cells (FDR $<0.1 \%$ ), mediated as a result of fusion-driven paracrine induction of EGFR signaling ${ }^{22}$ (Fig. 3c). Following manual curation, most associations between fusions and drug sensitivity could be readily explained by known interactions $(n=66 ; 30 \%)$, mutations in secondary genes $(n=7$; $3 \%)$, and fusions that were either not in frame $(n=77 ; 34 \%)$ or not detected in patient samples $(n=131 ; 57 \%)$. The remaining associations $(n=35 ; 15 \%)$ generally involve poorly described fusions present in two or three cell lines, making drug sensitivities difficult to interpret. This analysis suggests that besides wellestablished oncogenic fusions, there are few recurrent gene fusions that are associated with differential drug sensitivity, and which could be used as biomarkers for repurposing of existing anti-cancer drugs. We did, however, observe potent sensitivity to specific drugs in individual cell lines with rare fusions.

Functional fusion analysis using CRISPR-Cas9 loss-of-fitness data. Our analysis of fusions using drug sensitivity data was limited by their low frequency and the limited number of targets covered by available drugs. Here, we complemented our fusion identification pipeline with CRISPR-Cas9 screens to systematically assess fusion function based on their requirement for cell fitness. To be as comprehensive as possible, we considered all fusions including in-frame and not in frame transcripts. We assembled CRISPR-Cas9 whole-genome drop-out screening data from Project Score at the Sanger Institute ${ }^{23}$, Broad Institute DepMap project ${ }^{24}$, and Wang et al. ${ }^{6}$, which together span 371 cell lines from 33 different cancer types ${ }^{6,24}$. CRISPR loss-offitness screens typically target each gene with 5-10 single guide RNAs (sgRNAs) and average their fold changes to calculate genelevel depletion values. By contrast, we took advantage of individual sgRNA fold changes to query the functional importance of gene regions. We mapped the coordinates of the sgRNAs targeting either of the fusion genes, and classified them as mapping or non-mapping sgRNAs, depending on whether they targeted the fusion transcript or not (Fig. 4a). For each gene fusion, we calculated a fusion essentiality score (FES) representing the average difference between the mapping and non-mapping sgRNAs normalized and scaled fold changes (a measure of the cell fitness effect). For transcripts where there were only mapping guides (50\% of transcripts), the value of non-mapping guides was set to zero, allowing us to compare the effect in a specific cell line to the average effect across all cell lines. The statistical significance of each FES and FDR were calculated based on 10,000 randomizations of all sgRNA fold changes in the cell lines, and we set a minimum fold change of -0.45 between mapping and nonmapping sgRNA (see Methods).

We identified mapping sgRNA for 2821 (26\%) fusion transcripts, of which only 99 (4\%; representing 103 fusion events) were significantly associated with decreased cell fitness in at least one CRISPR dataset (FES FDR <5\%; Fig. $4 \mathrm{~b}$ and Supplementary Data 8 ). This corresponded to one or more functional fusions in $55(16 \%)$ tested cell lines. Using gene-set enrichment analysis (GSEA), functional fusions were enriched for transcripts reported in the COSMIC database of oncogenic fusions $(p<0.001$, GSEA), which included well-known fusions such as EML4-ALK, EWSR1FLI1, and KMT2A-MLLT3 and TPM3-NTRK1 (Fig. 4b, c and Supplementary Fig. $3 \mathrm{~b}$ ). In addition, amongst the most significant 
a

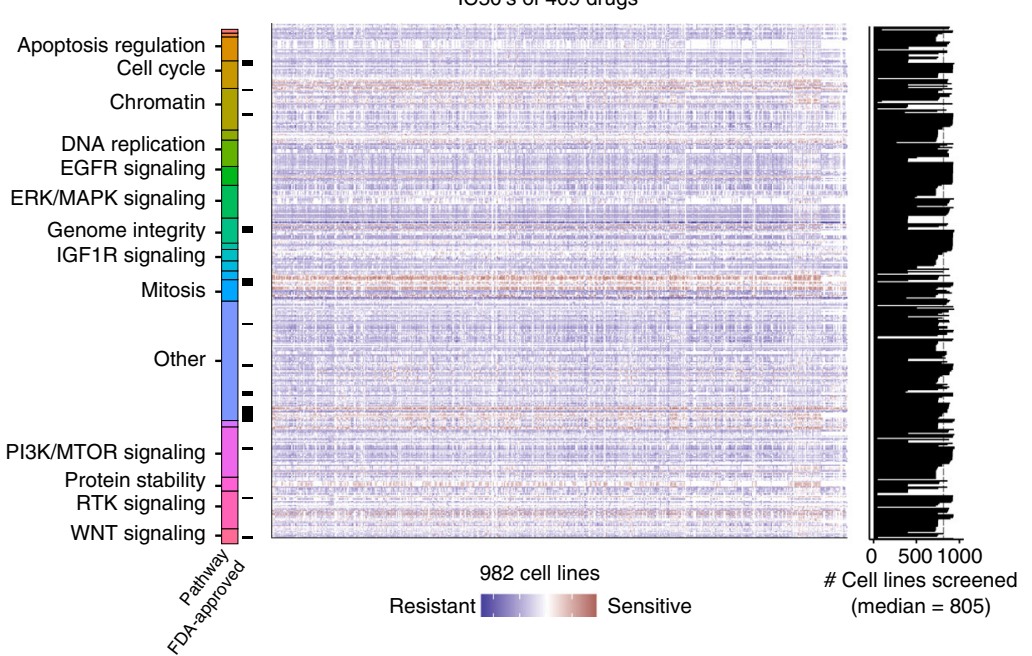

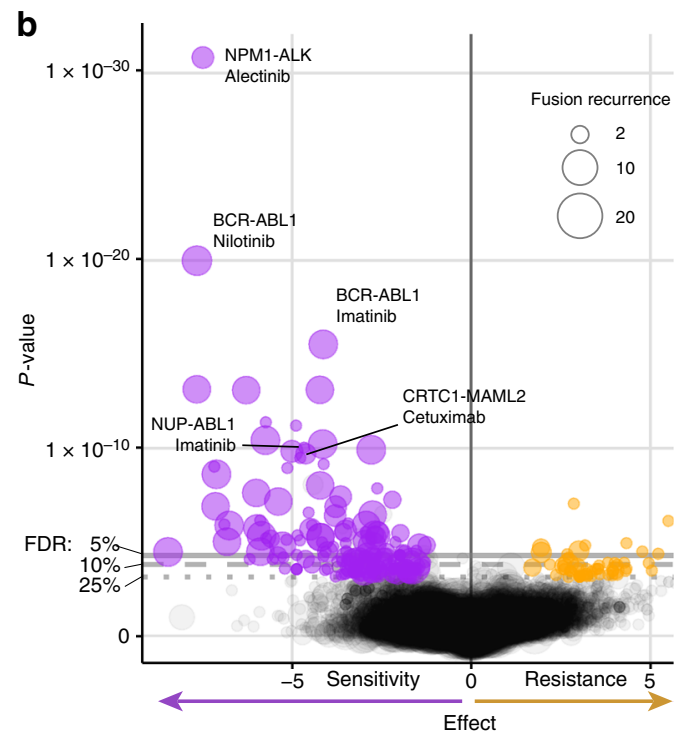
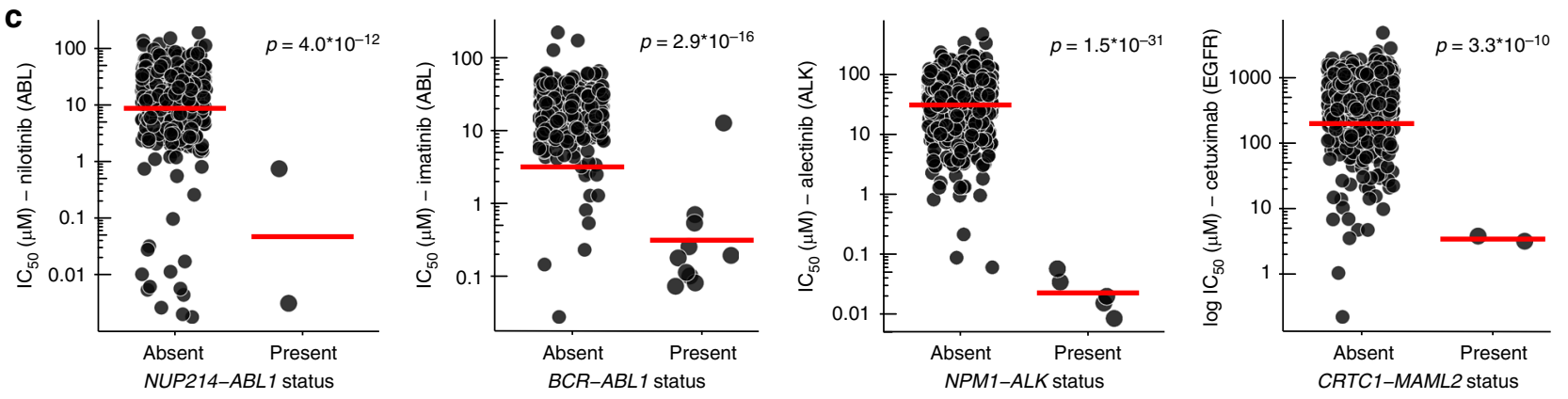

Fig. 3 Gene fusions as therapeutic biomarkers. a Genomics of Drug Sensitivity in Cancer (GDSC) drug sensitivity data utilized (reported as half-maximal inhibitory concentration ( $\mathrm{IC}_{50}$ ) values) with Food and Drug Administration (FDA)-approval status of compounds. Compounds are grouped by target or pathway. $\mathbf{b}$ Analysis of variance (ANOVA) results for fusion-drug associations. Each circle represents a tested association, with circle size indicating the number of cell lines harboring the associated fusion event (fusion recurrence), false discovery rate (FDR) thresholds are indicated. Negative effect sizes are associated with sensitivity and positive effect size resistance. Representative fusion-drug associations are labeled. c Examples of differential drug sensitivity in cell lines stratified by fusion status. Nominal therapeutic drug targets are in brackets. Each circle is the $\mathrm{IC}_{50}$ for an individual cell line and the red line is the geometric mean. Association significance values ( $p$ values) are from the ANOVA test

functional fusions were YAP1-MAML2 fusions (FDR $<0.01 \%$ ), DDX6-FOXR1 (FDR <0.5\%), and PICALM-MLLT10 (FDR $<0.5 \%)$. There was an enrichment in functional fusions for transcripts linked with drug sensitivity based on our ANOVA analysis ( $p<0.001$, GSEA; Fig. 4 b). Interestingly, there was no enrichment for fusion transcripts that were: (i) previously reported in patient samples; (ii) fusion transcripts that are in frame vs. not in frame; (iii) fusion transcripts in amplified regions, which are associated with non-specific fitness effects in CRISPR screens; ${ }^{25}$ (iv) nor for fusion transcripts that involve genes in the COSMIC Census ${ }^{26}$. Moreover, when considering only the subset of fusions most likely to be clinically relevant, namely those involving an in-frame event with a COSMIC oncogene, we did not observe an enrichment in functional fusions (Fig. 4b). Overall, for most tested fusions, we did not detect evidence supporting a functional role in cancer cell fitness.

Function of oncogenic gene fusions across different histologies. Despite an absence of evidence supporting the function of most fusions, as exemplified in the following analyses, we provide new insights into the pathogenic role of specific gene fusions that point to strategies for repurposing clinically approved drugs in rare subsets of fusion-positive cancers.

Rare RAF1 fusions occur in patient tumours ${ }^{27-29}$ and are biomarkers of response to mitogen-activated protein kinase pathway inhibition. We identified an in-frame $A T G 7-R A F 1$ fusion in PL18, a pancreatic adenocarcinoma cell line (Fig. 5a). The fusion was confirmed by Sanger sequencing and FISH (Fig. 5b and Supplementary Fig. 4a). The fusion removes the Nterminal regulatory region, but retains an intact $R A F 1$ protein kinase domain, suggesting it results in constitutive kinase activation. Only mapping sgRNAs targeting the portion of the two genes involved in the fusion were significantly depleted in PL18, resulting in a significant FES (Fig. 5c). Moreover, ATG7fusion-targeting sgRNA were only depleted in PL18 cells and not other pancreatic cell lines (Fig. 5d). Unlike $>90 \%$ of pancreatic tumours and cell lines that have activating mutations in KRAS $^{8,30}$, PL18 has a wild-type KRAS allele, but retained potent sensitivity to downstream MEK (mitogen-activated protein kinase kinase) pathway inhibitors trametinib and PD0325901 (Fig. 5e and Supplementary Fig. 4b). An ATG7-RAF1 rearrangement was previously reported in another KRAS wild-type pancreatic cancer model $^{31}$. Furthermore, we mined sequencing data for 126 pancreatic adenocarcinoma patient-derived xenograft (PDX) models and identified an additional KRAS wild-type tumour with a PDZRN3-RAF1 fusion, which conserves the RAF1 kinase domain (Supplementary Fig. 4f). Together, our analysis supports emerging evidence for rare recurrent and potentially therapeutically actionable $R A F 1$ rearrangements in KRAS wild-type pancreatic cancer. 
a

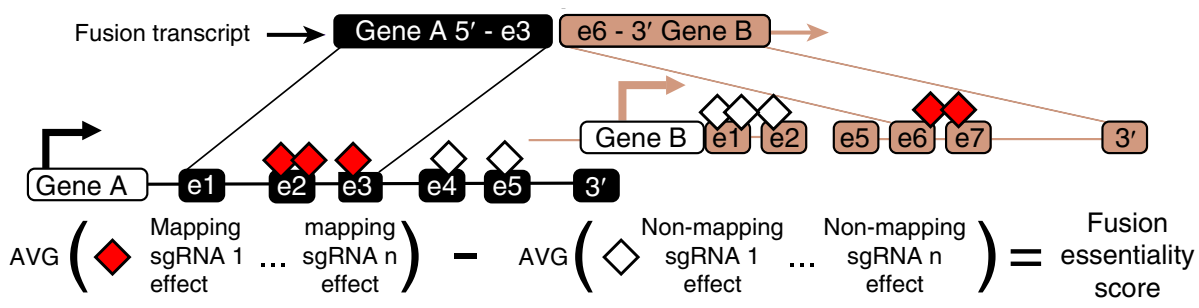

b

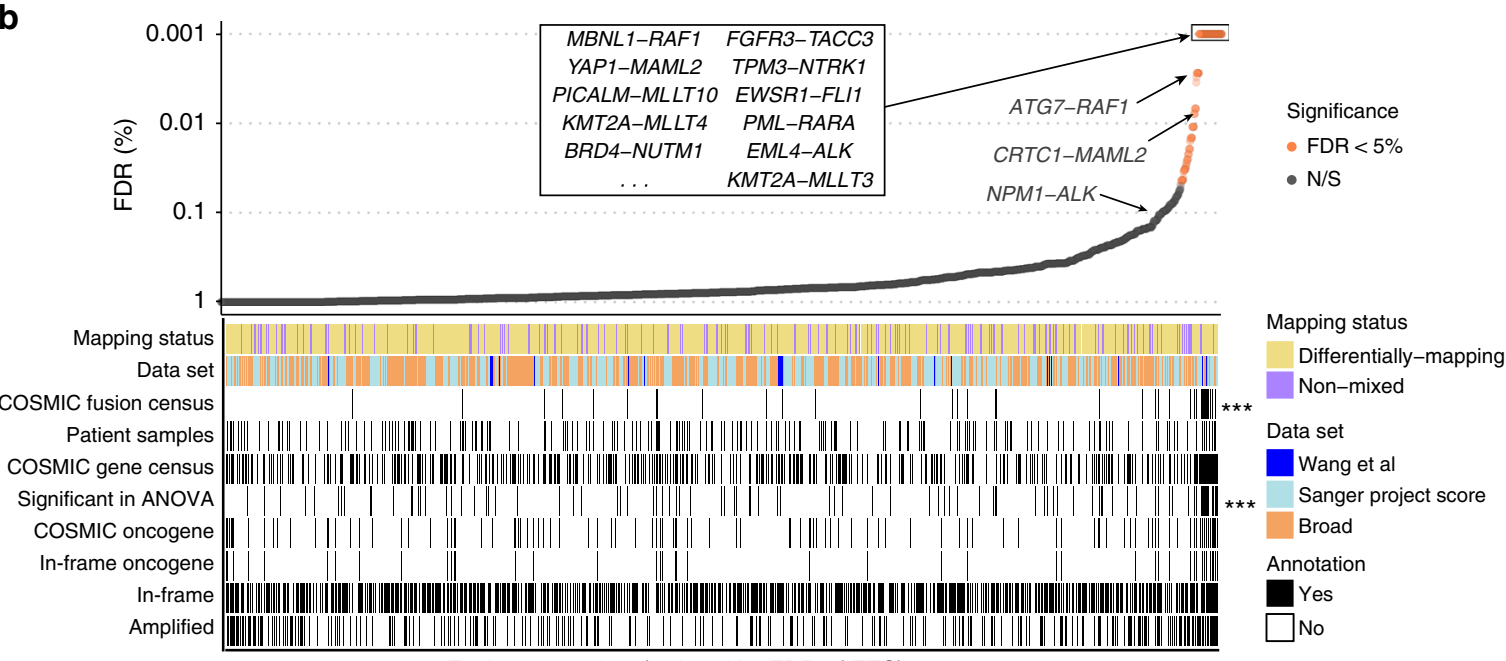

Fusion transcripts (ordered by FDR of FES)

C
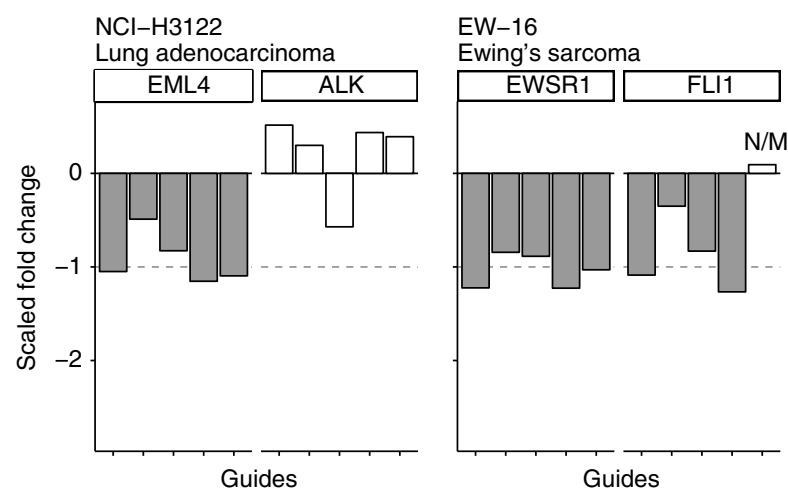

MOLM-13

Acute myeloid leukaemia

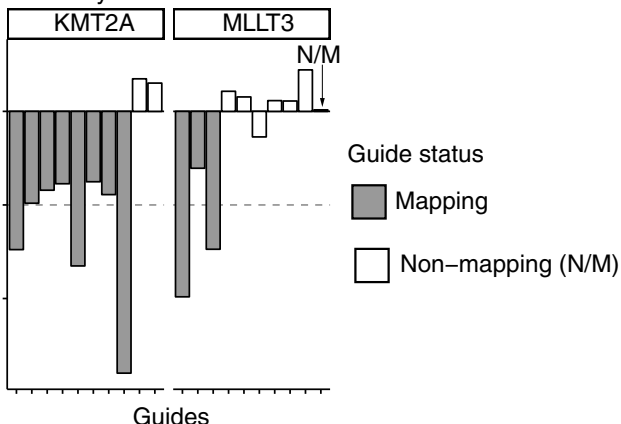

Fig. 4 CRISPR screening data identifies functional fusions. a Calculation of fusion essentiality scores (FES) by measuring the differential fitness effect of mapping vs. non-mapping single guide RNAs (sgRNAs) to each gene in a fusion transcript. b False discovery rate (FDR) of FES scores for all testable fusion transcripts $(n=2821)$. Transcripts with at least one mapping and one non-mapping guide are "differentially mapping," while transcripts with only mapping guides are "non-mixed". Fusion transcripts were classified into the indicated genes sets and $p$ values were calculated using gene-set enrichment analysis (GSEA). Selected known oncogenic fusions and other fusions of interest are labeled. c Examples of functional fusion transcripts identified in specific cancer cell lines based on FES scoring. Each bar is the scaled fold change of an individual sgRNA to fusion $5^{\prime}$ and $3^{\prime}$ end partner genes, and colored by fusion mapping or non-mapping sgRNA. Dashed line is at -1 (to which known essential guides were scaled). AVG = average; N/S = not significant at $5 \%$ FDR

BRD4-NUTM1 fusions genetically define NUT midline carcinoma (NMC), a rare and aggressive neoplasm that usually arises in the midline of the body with marked sensitivity to BET bromodomain inhibitors ${ }^{32,33}$. We identified a novel inframe BRD4-NUTM1 fusion in SBC-3, a cell line established from a 24-year-old male diagnosed with small-cell lung carcinoma (SCLC) $^{34}$, and confirmed the fusion by Sanger sequencing and FISH (Fig. 5a, b and Supplementary Fig. 4a). Based on CRISPR data for 206 cell lines screened at Sanger, NUTM1targeting guides were highly depleted only in SBC-3 cells, and the fusion was associated with a significant FES (Fig. $5 \mathrm{c}$ and Supplementary Fig. 4c). Moreover, SBC-3 cells displayed marked sensitivity to four different BET inhibitors (Fig. 5e and Supplementary Fig. 4b).
We investigated whether the SBC-3 cell line is mis-classified as an SCLC and is actually a rare NMC of the lung. Unlike $>95 \%$ of SCLC tumours and cell lines, SBC-3 cells do not have alterations in $R B 1$ or TP53, nor do they express SCLC-specific neuroendocrine markers, such as CgA, NSE, and synaptophysin (Supplementary Fig. 4d). The BRD4-NUTM1 fusion was specifically associated with high NUTM1 transcript expression in cell lines (Fig. 2b and Supplementary Fig. 4e). Therefore, we mined The Cancer Genome Atlas (TCGA) expression data for SCLC and non-SCLC (NSCLC) searching for samples displaying high NUTM1 mRNA levels. We identified a single NSCLC sample derived from a 39-year-old patient diagnosed with lung squamous cell carcinoma, displaying NUTM1 mRNA outlier expression (Supplementary Fig. 4e) and carrying a NSD3-NUTM1 
a
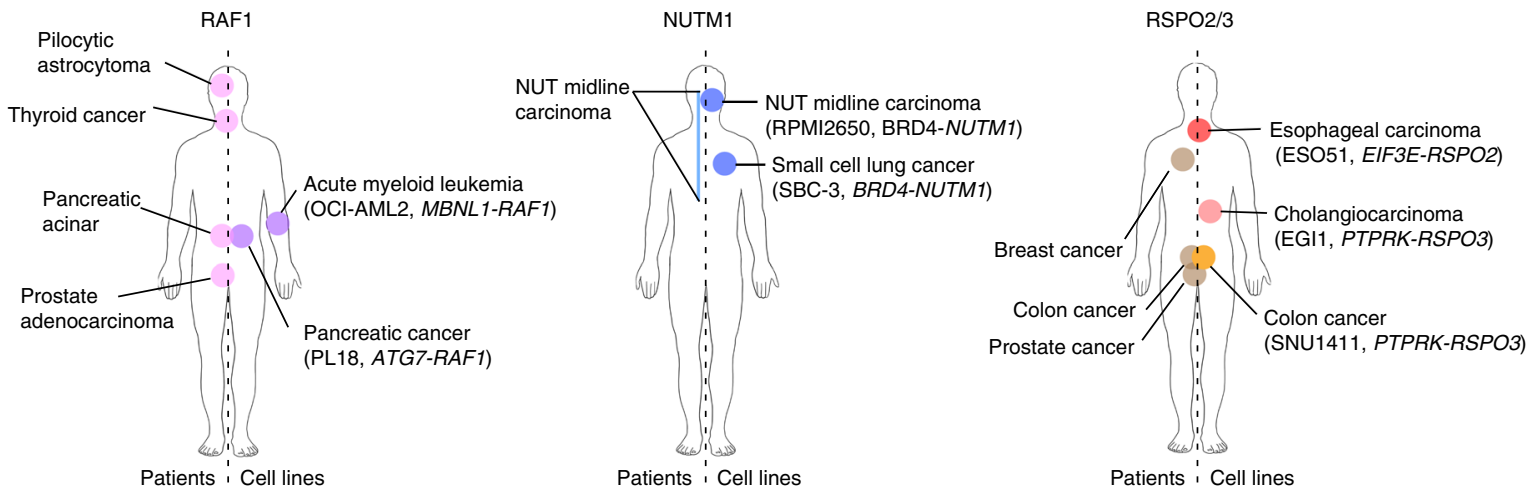

b
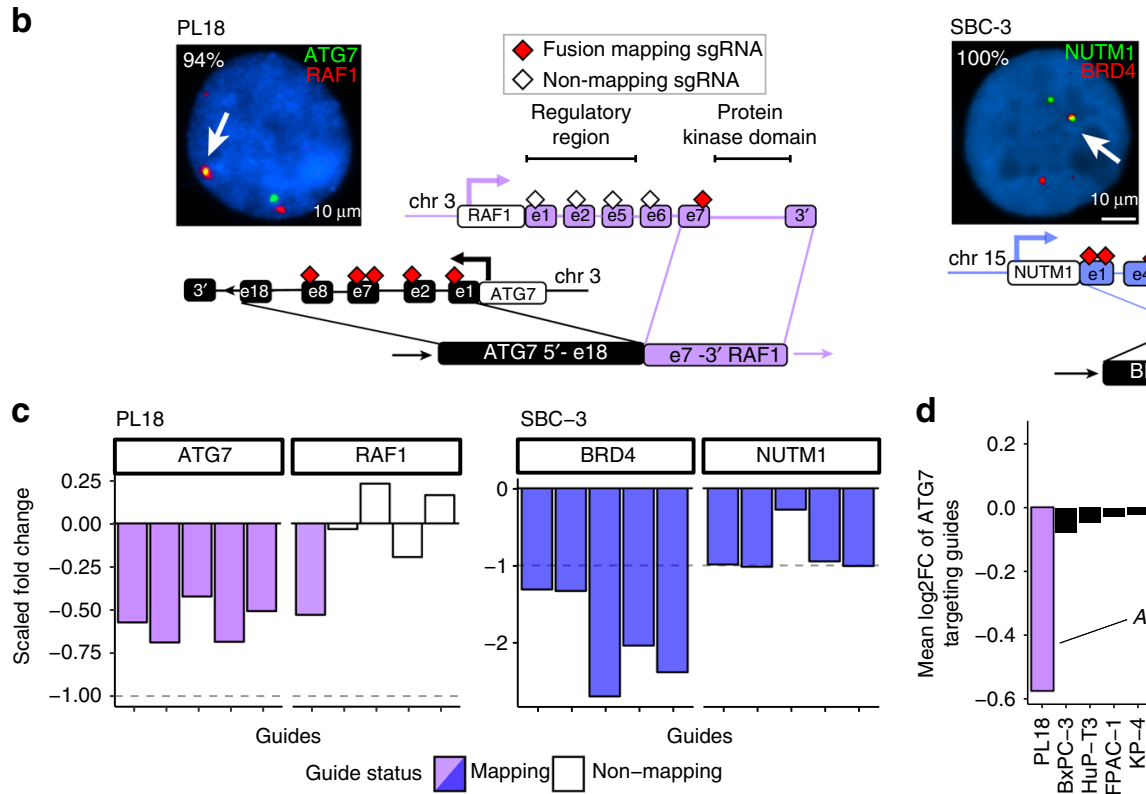

Patients : Cell lines

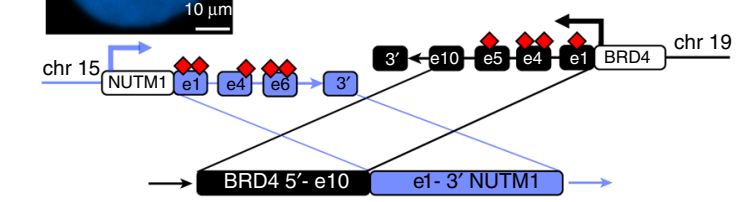

e
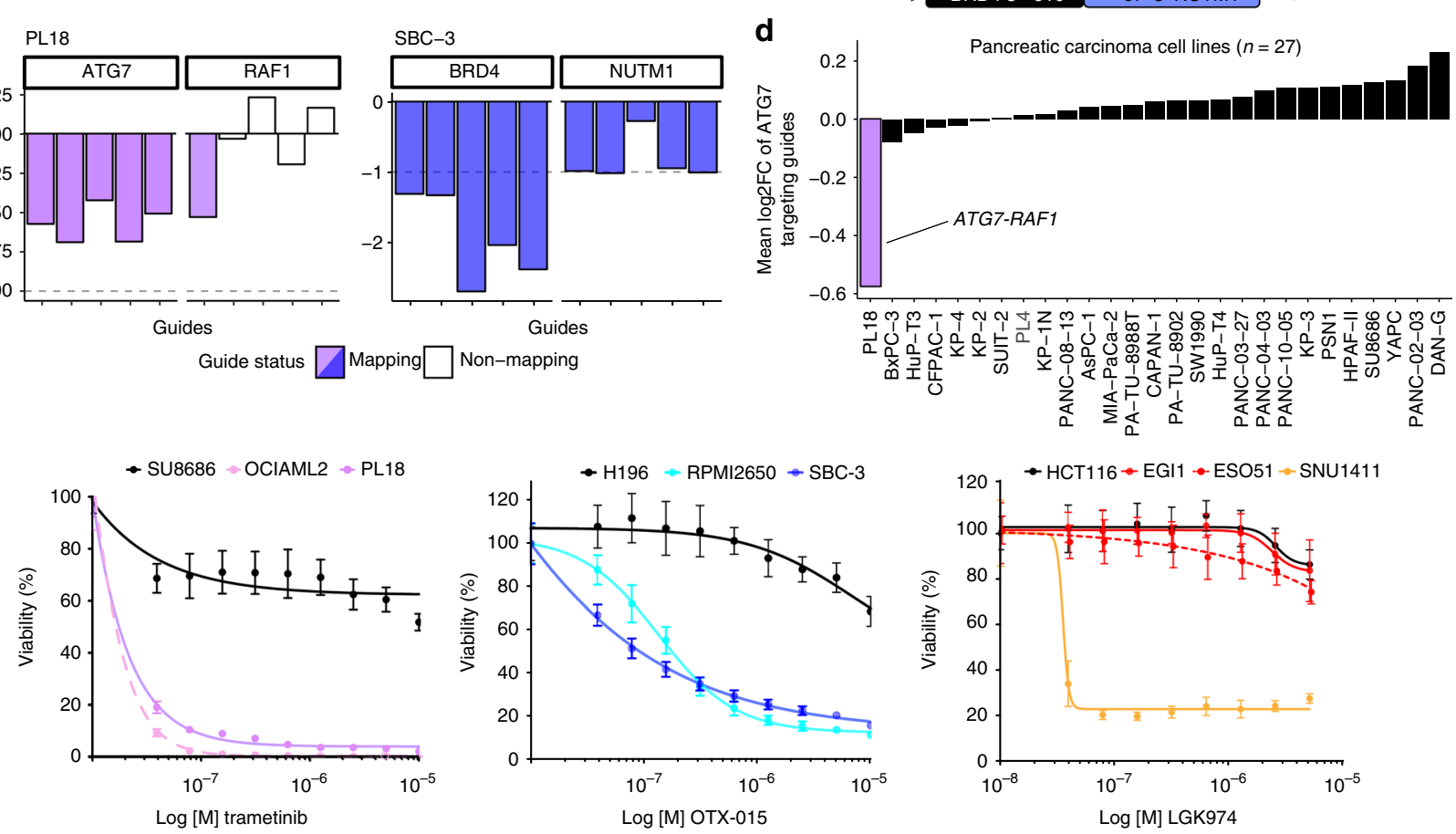

Fig. 5 Therapeutically actionable oncogenic fusions identified across different histologies. a RAF1, NUTM1, and RSPO2/3 fusions identified in patients previously (left) and cell lines in this study (right). Cell lines with known oncogenic fusions used as positive controls are reported. b Interphase fluorescence in situ hybridization (FISH) of ATG7-RAF1 (left) and BRD4-NUTM1 (right) gene fusions (arrows) in PL18 and SBC-3 cell lines. The percentage of fusion-positive interphases are reported in white text. Schematic representations of each fusions are represented. Only exons involved in the breakpoint or displaying fusion mapping single guide RNA (sgRNAs) (red diamonds) or non-mapping sgRNAs (empty diamonds) are shown. c Fold change fusion essentiality score (FES) values of sgRNAs targeting ATG7 and RAF1 in PL18 (left) and BRD4 and NUTM1 in SBC-3 cells (right). Colored bars indicate values of sgRNAs targeting the exons involved in the fusions. d Depletion of fusion-targeting ATG7 guides for all screened pancreatic cancer cell lines. e Viability assay on PL18, SBC-3, EGI-1, and ESO51 cells treated with MEK (trametinib), BET (OTX-015), and PORCN (LGK974) inhibitors, respectively. SU8686, H196, and HCT116 cells are pancreatic, small-cell lung cancer, and colorectal cancer-negative controls. OCIAML2, RPMI2650, and SNU1411 are, respectively, a RAF1-rearranged leukemia, a NUTM1-rearranged NUT midline carcinoma (NMC), and a RSPO3-rearranged CRC cell line included as positive controls. Data are average \pm s.d. of three technical replicates and are representative of three independent experiments

rearrangement ${ }^{15}$ (Supplementary Fig. 4f), a chimeric oncoprotein recently identified in NMC patients and associated with BET inhibitor sensitivity ${ }^{35}$. NUT rearrangements occur in a rare subpopulation of patients diagnosed with SCLC and NSCLC ${ }^{36,37}$, and recent studies established that NUT-associated fusions can occur in tumours outside the midline, such as soft tissue, brain, and kidney ${ }^{38}$. Thus, although we cannot exclude misclassification of SBC-3 cells, our preclinical data support evidence that 
functional NUTM1 fusions are present in tumours diagnosed as lung cancer, and could represent an actionable driver event in these tumours with immediate potential clinical implications.

Aberrant expression of RSPO2/3 fusion transcripts synergize with WNT ligands to trigger WNT pathway activation in APC wild-type colorectal cancer $(\mathrm{CRC})^{16}$. WNT pathway blockade with porcupine inhibitors is effective in RSPO3-rearranged CRC preclinical models $^{39,40}$ and clinical trials in patients with RSPO2/3-fusionpositive tumours of any histological origin are ongoing (NCT01351103). Here, we detected and validated two unreported canonical R-spondin fusions in cancer cell lines derived from biliary tract (EGI-1; PTPRK-RSPO3 fusion) and esophagus (ESO51; EIF3ERSPO2 fusion) by PCR and FISH (Fig. 5a and Supplementary Fig. $5 \mathrm{a}, \mathrm{b}$ and c). Aberrant expression of $\mathrm{RSPO} 2 / 3$ was detected in both cell lines (Fig. $2 \mathrm{~b}$ and Supplementary Fig. 5d). Similarly, through mining TCGA esophageal cancer data, we found that a tumour with high $\mathrm{RSPO} 3$ expression was positive for a canonical RSPO3 fusion $^{15}$ (Supplementary Fig. 5d). Surprisingly, EGI-1 and ESO51 were insensitive to WNT pathway blockade with a porcupine inhibitor (Fig. 5e and Supplementary Fig. 5e). This was in contrast to SNU1411, a positive control CRC cell line model addicted to WNT pathway activation by rearranged RSPO3, which was sensitive to multiple porcupine inhibitors ${ }^{41}$. This result points to an element of tissue specificity in mediating the functional role of some fusions, with potentially important implications for repurposing of WNT pathway inhibitors across different RSPO-fusion-positive tumour types. Interestingly, sgRNA mapping to the $R S P O 2 / 3$ fusion were not associated with significant FES in EGI-1 and ESO51, nor in the positive control line SNU1411 (Supplementary Fig. 5f-h). This may be because RSPO2/3 fusions act as paracrine signals to enhance WNT ligands and so are not readily detected by pooled CRISPR screens designed to unveil cell intrinsic gene dependencies.

Recurrent YAP1-MAML2 fusions drive Hippo pathway signaling. We next investigated the function of a poorly understood fusion. Recurrent YAP1-MAML2 fusions were identified in AM38 (glioblastoma), ES-2 (ovarian carcinoma), and SAS (head and neck carcinoma) cell lines (Fig. 6a). We validated the fusion in all three cell lines by PCR, and interphase and fiber FISH (Fig. 6b, c and Supplementary Fig. 6a). YAP1-MAML2 fusions have been reported in nasopharyngeal carcinomas ${ }^{42}$ and in a sample from a patient with skin cancer, but not in the three tumour types reported here ${ }^{15}$ (Fig. 6a). The fusion brings together exons $1-5$ of YAP1 and exons 2-5 of MAML2, a transcript structure that is conserved across all cell lines and patient samples (Fig. 6d and Supplementary Fig. 6b).

A functional role for YAP1-MAML2 fusions has not been reported. We found that YAP1-MAML2 fusions were significantly associated with decreased cell fitness when targeted in the CRISPR screen (Figs. 4b, 6e). Furthermore, loss of fitness in response to MAML2 knockout is unique to MAML2-fused cell lines in the three cancer types where the fusion is observed (Fig. 6f). YAP1 aberrant activation is linked with poor prognosis, chemoresistance, and resistance to cell death in multiple solid tumours ${ }^{43,44}$. YAP1 is a transcriptional co-activator of the Hippo pathway through binding with the TEAD1 transcription factor and MAML2 is a transcriptional co-activator involved in NOTCH signaling 45 . YAP1-MAML2 fuses the transcriptional activation domain of MAML2 with the TEAD-binding domain of YAP1. Intriguingly, ES-2 and AM-38, although not SAS, also showed essentiality for TEAD1 in the CRISPR drop-out screen (Supplementary Fig. 6d), suggesting that the fusion protein signals through TEAD1.

To further investigate fusion protein activity, we performed GSEA comparing the three YAP1-MAML2-fusion-positive cell lines against all others. Of 189 pathways tested, a YAP1-conserved transcriptional signature was the most significant hit (adjusted $p<0.001$; Fig. $6 \mathrm{~g}$ ). The same signature was highly enriched when ES-2 was compared against all other ovarian cancer cell lines and SAS against all other head and neck cell lines, while expression of prototypic tissuespecific oncogenic signatures, such as estrogen receptor signaling in ovary, were depleted (Supplementary Fig. 6e). Overall, our findings that recurrent YAP1-MAML2 fusion are associated with increased YAP1 signaling and required for cell fitness support targeting the Hippo signaling cascade in YAP1-MAML2-fusion-positive tumours.

\section{Discussion}

Thousands of gene fusion transcripts have been reported $15,46,47$, and most are likely to be passenger events due to chromosomal instability or artifactual. We developed a multi-algorithm fusion calling pipeline, and integrated large-scale genomic and functional datasets, including CRISPR-Cas9 screening data, to systematically identify functional gene fusions across diverse tissue histology. Our analysis is a valuable reference of gene fusions in cancer cell lines. Furthermore, since fusions can have diagnostic, prognostic, and therapeutic utility, our analysis could have clinical implications.

Using our analysis pipeline, we tested 3354 fusion events and found supporting evidence of a functional role for $368(11.8 \%)$ by either CRISPR data $(n=103)$ or drug sensitivity analysis $(n=$ 284) (Fig. 6h). Thus, most fusions are likely to be passenger events and dispensable for cell fitness. Of those with functional evidence, only $142(38.5 \%)$ involved a COSMIC cancer gene, 58 (16\%) were listed in the COSMIC fusion census, and 107 (29\%) were called in TCGA patient samples. Thus, many fusions with supporting functional evidence are poorly understood and do not contain known driver genes, indicating that there are gaps in our knowledge of genes with roles in cancer cell fitness.

Although our analysis suggests that most fusions are not functional, there are several limitations to our approach. Some fusions may be required for aspects of the malignant phenotype not measured here, such as tumour initiation, paracrine signaling, host-tumour cell interaction, and metastasis. Our CRISPR-based approach is only suitable for testing fusions with mapping sgRNA, and is subject to possible bias based on sgRNA efficiency and the number of mapping vs. non-mapping sgRNA. Furthermore, this approach captures fusions that induce gain-of-function or dominant-negative effects, but is not able to identify loss-of-function effects, such as inactivation of a tumour suppressor. Some tumour types are relatively poorly represented, including hematological and pediatric malignancies, and it may be that in some tumour types we observe different patterns of functionality. Finally, sub-clonal fusions could lead to false-negative results. Despite these limitations, our finding that most fusions tested do not have supporting functional evidence, including fusions with cancer drivers genes, emphasizes the importance of analyses to ascribe function when interpreting fusions identified using genomic sequencing.

Gene fusions are used as therapeutic biomarkers to enroll patients in clinical trials and to direct clinical care, often in diverse histologies and clinico-pathologic subtypes. Notable examples are NTRK and ALK fusions, originally identified as effective biomarkers of response to targeted agents in NSCLC patients and occurring at low frequencies $(<1 \%)$ in a variety of malignancies ${ }^{48-50}$. We provide specific and previously undescribed data on fusions involving RAF1, ROS1, and BRD4 that suggest that existing drugs could be repurposed for use in rare pancreatic, breast, and lung cancers. FISH analysis confirmed the presence of these gene fusions in the vast majority of the cell nuclei analyzed $(>90 \%)$, indicating that these alterations are clonal events. Further studies using tumour xenograft models would support the in vivo efficacy of these findings and could 
a

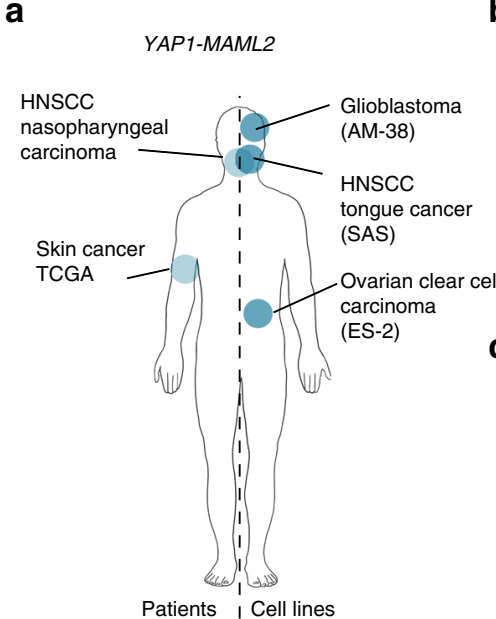

b

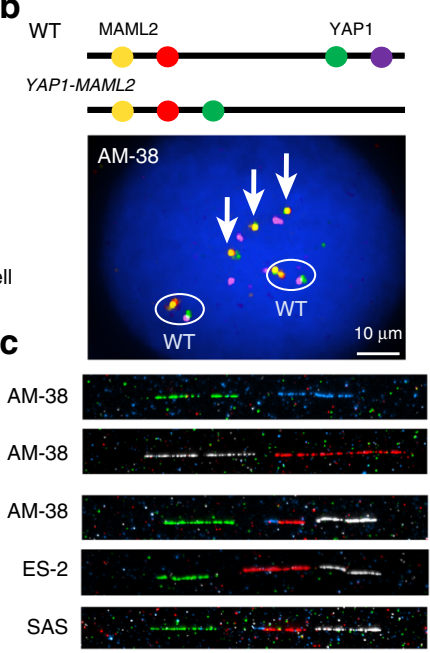

d

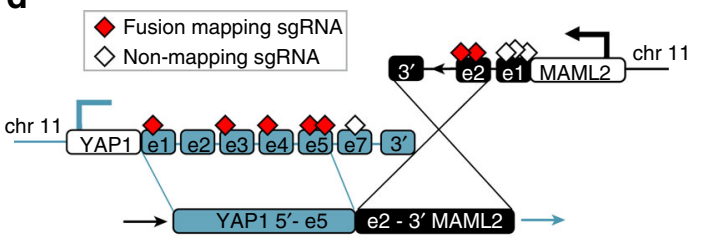

e

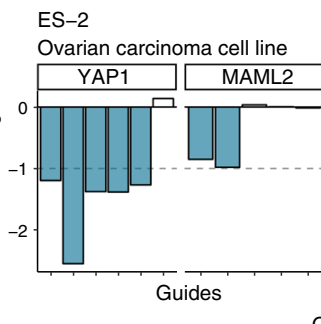

AM-38

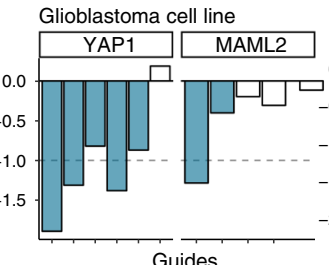

SAS

Head and neck cell line

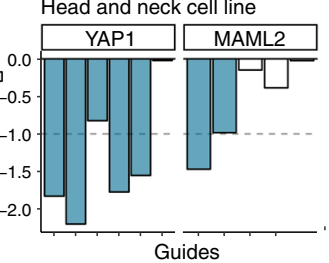

Guides

YAP1-MAML2

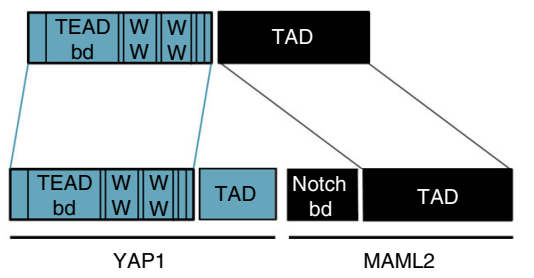

f

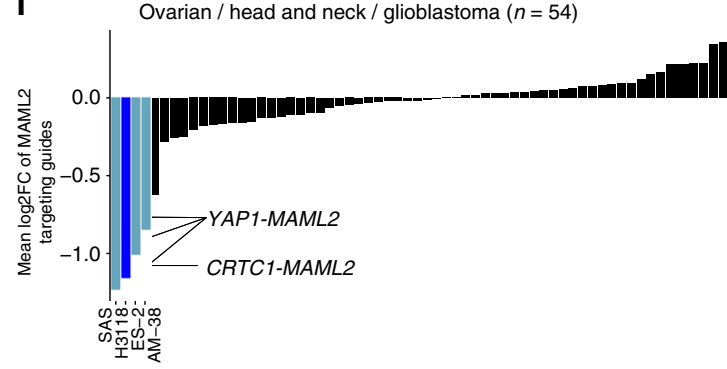

g

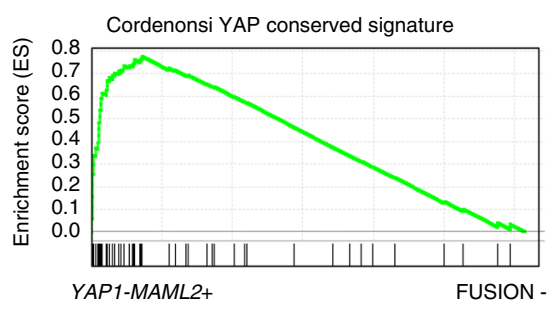

h

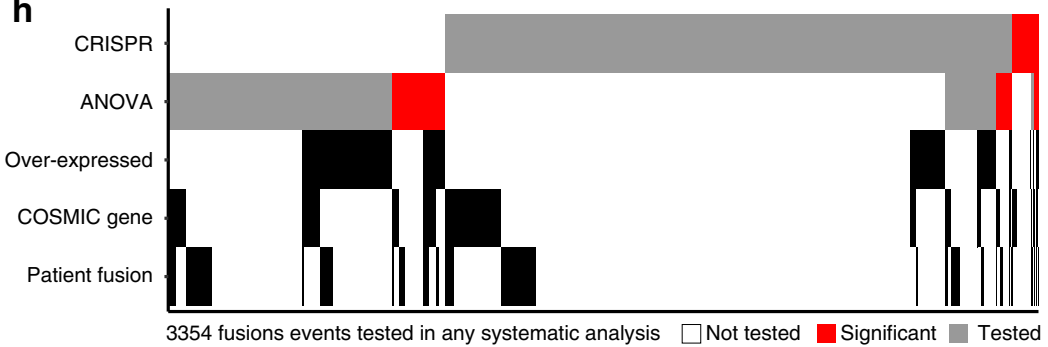

Fig. 6 Recurrent YAP1-MAML2 fusions activate Hippo pathway signaling. a YAP1-MAML2 fusions in patient tumours (left) and cell lines (right). b Interphase fluorescence in situ hybridization (FISH) (AM-38 only) targeting YAP1-MAML2 fusion (arrows; cells are polyploid with wild-type chromosomes circled) in AM-38, ES-2, and SAS cell lines. Probes used and chromosomal position are shown schematically. YAP1 and MAML2 are both on chromosome 11. c Fiber FISH showing gene fusion in AM-38, ES-2, and SAS cell lines. MAML2 probes are shown in green and blue; YAP1 probes are shown in white and red. $\mathbf{d}$ Schematic of YAP1-MAML2 and functional domains involved in the fusion. e Fold-change values of single guide RNAs (sgRNAs) targeting YAP1 and MAML2 genes in ES-2, AM-38, and SAS cell lines. $\mathbf{f}$ YAP1-MAML2 fusion-positive cell lines show the highest depletion of fusion-targeting MAML2 guides in ovary, head and neck, and glioblastoma cell lines $(n=54)$. Cell line in dark blue (H3118) harbors a known CRTC1-MAML2 fusion (Fig. 3c) ${ }^{22}$. g GSEA of YAP1 gene signature in YAP1-MAML2-positive cells $(n=3)$ vs. fusion-negative $(n=1008)$ cell lines. $\mathbf{h}$ Heatmap of 3345 fusion events tested in CRISPR and analysis of variance (ANOVA) systematic analyses. Fusions events are annotated if one of the partner genes is significantly overexpressed using our linear regression model, contain a COSMIC cancer driver gene, or has been detected in patient samples

pave the way for their clinical application. More broadly, these results support the use of validated oncogenic fusions as therapeutic biomarkers in diverse histologies, and the utility of basket trials for clinical development of drugs targeting fusion proteins irrespective of tumour type, such as the type used for the development of entrectinib in solid tumours with $A L K, R O S 1$, and $N T R K$ fusions ${ }^{50}$. A notable exception in our analysis was the differential sensitivity to WNT pathway inhibition of CRC vs. biliary tract and esophageal cancer cell lines with canonical Rspondin fusions. This suggests that tissue context could impact the functional role of some fusions as has been observed for oncogenes (e.g., BRAF-mutated $\mathrm{CRC}^{51}$ ). Further investigations are warranted to understand this difference, and drug combinations could be evaluated in these specific context to overcome resistance similar to what is in clinical development for BRAFmutated $\mathrm{CRC}^{51}$.
We identified and functionally evaluated less well-studied gene fusions, as exemplified by YAP1-MAML2 rearrangements, which are required for cell fitness in multiple histology and associated with increased YAP1 signaling. Given the emerging role of YAP1/ TEAD1 and the Hippo pathway in cancer, there is interest in pharmacological inhibition of Hippo signaling as an anti-cancer therapeutic strategy ${ }^{52}$. We provide preclinical evidence supporting inhibition of this signaling axis in YAP1-MAML2-fusionpositive tumours, which could pave the way for clinical development in a rare but defined patient population.

In summary, our findings that most fusions tested were dispensable for tumour cell fitness has implication for the interpretation of fusions detected in tumour sequencing data. Furthermore, this observation supports the use of systematic functional studies in preclinical models as an unbiased platform to systematically assess the impact of fusions in cancer. Extending this approach to a larger 
set of cancer models that represents the histopathologic and genomic diversity of patient tumours could reveal additional new insights with clinical relevance. Notably, here we identified fusion drivers of carcinogenesis, which could represent targets for drug development and specific actionable leads with potential for immediate clinical development in defined fusion-positive patients.

\section{Methods}

Sample selection. A collection of 1011 cancer cell lines have been compiled from publicly available repositories as well as private collections and maintained following supplier guidelines. These cell lines were selected to be genetically unique based on short tandem repeat (STR) and single-nucleotide polymorphism (SNP) fingerprints (http://cancer.sanger.ac.uk/cell_lines/download). STR profiles matched those in public repositories or match published STR profiles. The cells lines have been extensively characterized using whole-exome sequencing

(EGAD00001001039), Affymetrix SNP6-based copy number analysis, and genotyping (EGAD00010000644). The origin of all cell lines and related data are available through the Cell Model Passports database (https://cellmodelpassports. sanger.ac.uk/ $/)^{9}$. All cell lines have been tested for mycoplasma using a PCR-based assay (EZ-PCR Mycoplasma Detection Kit, Biological Industries) and a biochemical test (MycoAlert, Lonza). Cell lines that test positive using either method are removed from the collection. Comparing SNP6 genotyping data and somatic variant data, we verified that cell lines overlap extensively with those characterized by the Cancer Cell Line Encyclopedia (TCGA). See Supplementary Data 1 for a complete description of the cell lines and their molecular annotation. This study includes the following commonly misidentified lines: TE-12, NCI-H1304, MELHO, U-118-MG, BT-549, BE-13, ETK-1, GT3TKB, MKN28, RPMI-6666, SK-MG1, CGTH-W-1, H513, OVMIU, and KP-1N. Misidentified lines have been noted in Supplementary Data 1 and on the Cell Model Passport (https://cellmodelpassports. sanger.ac.uk). Mis-identification does not impact tissue of origin, genomic data used for analyses, or results. SNU1411 were purchased from KCLB and maintained in RPMI- 1640 with L-glutamine $(300 \mathrm{mg} / \mathrm{L}), 25 \mathrm{mM} \mathrm{HEPES}$ and $25 \mathrm{mM} \mathrm{NaHCO}_{3}$, $90 \%$; heat-inactivated fetal bovine serum, $10 \%$.

RNA-seq data and identification of fusion transcripts. RNA-seq data for 589 cell lines was obtained from the CGHub, and 450 cell lines were sequenced at the Sanger Institute (EGAS00001000828). For 23 cell lines, sequence was obtained from both CGHub and Sanger Institute to allow comparison of the output based on the sequence from the two studies. Where replicated datasets were available, we took forward only fusions called from Sanger Institute sequencing data for our final analysis.

For sequencing performed at the Sanger Institute, cell line pellets were collected during exponential growth in RPMI or Dulbecco's modified Eagle's medium/F12 and were lysed with TRIzol (Life Technologies) and stored at $-70^{\circ} \mathrm{C}$. Following chloroform extraction, total RNA was isolated using the RNeasy Mini Kit (Qiagen). DNAse digestion was followed by the RNAClean Kit (Agencourt Bioscience). RNA integrity was confirmed on a Bioanalyzer 2100 (Agilent Technologies) prior to labeling using $3^{\prime}$ IVT Express (Affymetrix). Sequence libraries were prepared in an automated fashion on the Agilent Bravo platform using the stranded mRNA Library Prep Kit from KAPA Biosystems. Processing steps were unchanged from those specified in the KAPA manual, except for use of an in-house indexing set. Three publicly available gene fusion detection algorithms were used (TopHatFusion (v2.1.0), STAR-Fusion (v2.5.0), and deFuse (v0.7.0)) as described in GitHub (https://github.com/cancerit/cgpRna/blob/dev/README.md).

Fusion transcript filtering criteria. From the output of the three distinct fusion detection algorithms, we selected for analysis only fusions that were called with four or more reads that align directly across the breakpoint. We also required fusions to be called by at least two different algorithms. Next, we removed fusions identified from the analysis of 245 non-neoplastic samples downloaded from $\mathrm{GTEx}^{53}$.

Fusion annotations. The frame of fusion transcripts was predicted using the GRASS algorithm that is built into the fusion-calling pipeline (https://github.com/ cancerit/cgpRna/blob/dev/README.md/https:/github.com/cancerit/grass). A list of known cancer driver genes was obtained from the COSMIC cancer census in June 2017 (https://cancer.sanger.ac.uk/cosmic/curation). Known cancer fusions, as well as annotation of TSGs and oncogenes, were downloaded from the COSMIC fusion census (https://cancer.sanger.ac.uk/cosmic/fusion). Our reference set of fusions identified previously in patient samples comes from an analysis of fusions in over 9000 TCGA samples ${ }^{15}$.

PCR validation of fusion transcript. Complementary DNA (cDNA) was prepared using the Superscript double-stranded cDNA Synthesis Kit (Invitrogen) followed by SPRI (Solid Phase Reversible Immobilization) clean-up. The cDNA was then subjected to "whole-genome amplification" using the Illustra GenomiPhi HY DNA Amplification Kit as per the manufacturer's instructions. This WGA'ed cDNA was used as a template for the PCR validation. Generally two distinct sets of PCR primers were designed using Primer3 (http://www.bioinformatics.nl/cgi-bin/ primer3plus/primer3plus.cgi) for each fusion junction tested (Primer sequences are in Supplementary Data 9). The primers were then checked by ePCR (http://www. ncbi.nlm.nih.gov/sutils/e-pcr/reverse.cgi?taxi-

$\mathrm{d}=9606 \& \mathrm{db}=2 \&$ orgdb $=118 \&$ margin $=200 \&$ mism $=0$ \&gaps $=0$ ) against the genome and transcriptome to make sure that they would not produce a PCR product of $<5 \mathrm{~kb}$. PCRs were carried out in duplicate using two PCR programs: (1) 30 cycles, $95^{\circ} \mathrm{C}$ for $30 \mathrm{~s}, 60^{\circ} \mathrm{C}$ for $30 \mathrm{~s}$, and $72^{\circ} \mathrm{C}$ for $30 \mathrm{~s}$; (2) a touchdown program reducing annealing temperature by $2^{\circ} \mathrm{C}$ every two cycles, dropping from $60^{\circ} \mathrm{C}$ to $50^{\circ} \mathrm{C}$ over ten cycles, with a final 20 cycles at $50^{\circ} \mathrm{C}$. For all cycles, the melting and extension temperatures were $95^{\circ} \mathrm{C}$ and $72{ }^{\circ} \mathrm{C}$, respectively, and all stages were maintained for $30 \mathrm{~s}$. Finally, the PCRs were checked by gel electrophoresis to confirm the presence of a product of the predicted size. To validate candidate fusions, PCR products were further checked by PCR product Sanger sequencing. In these cases, the PCR products were first cleaned up using ExoSAP (Affymetrix) and then capillary sequenced by Eurofins Genomics (Ebersberg, Germany).

Differential fusion frequencies across cancer types. We designed an empirical permutation approach to identify genes with differential fusion frequencies across cancer types. Briefly, we built a binary matrix (genes $\times$ samples), where 1 indicates that the sample contains at least one fusion involving a gene $G$ and 0 indicates that the gene $G$ is not fused. Under the null hypothesis that gene alterations distribute homogeneously across cancer types (i.e., any sample from any cancer type has the same likelihood of having the gene $G$ fused), we permuted 10,000 times the initially observed matrix using the algorithm BiRewire implemented in an R package ${ }^{54}$. This algorithm generates randomized binary networks that preserve marginal totals. Randomized networks are used to build an empirical null distribution, modeling the likelihood of observing a gene fused in $X$ samples. Thus, we can derive a $p$ value per gene and cancer type representing the probability of observing $\geq N$ samples with the gene $G$ fused in the null distribution. Nominal $p$ values are adjusted using the FDR method.

Gene expression and differential gene expression analysis. Read counts per gene, based on the union of all exons from all possible transcripts, were used to calculate reads per kilobase per million (RPKM) as described previously ${ }^{10}$. To identify genes which expression is significantly altered when fused, we used MLR. For each fusion-associated gene, the expression values $G\left(\log _{2}\right.$ RPKM) from each sample $S$ are modeled as a function of the fusion status of the gene in the sample $S\left(X_{\text {fusion }}\right)$, a series of dependent covariates ( $X_{\text {covariates }}$, including cancer type in pan-cancer analyses, microsatellite instability status, and gene absolute CNA status), and a noise term $(\psi)$ :

$$
G=\beta_{\text {covariates }} X_{\text {covariates }}+\beta_{\text {fusion }} X_{\text {fusion }}+\psi \text {. }
$$

The association between the fusion status and gene expression was defined by the regression coefficient $\left(\beta_{\text {fusion }}\right)$ estimated with a multiple linear least-squares regression. Significance of the regressors was estimated with a type II ANOVA method from the car R package. For each cancer type, $p$ values were adjusted for multiple testing correction using the Benjamini-Hochberg method. To annotate fusion genes for overexpression of the $3^{\prime}$ gene, we selected fusions where the expression level of the $3^{\prime}$ gene is above the 95 th percentile (i.e., $95 \%$ of the cell lines have an expression level lower than the one observed in the cell line carrying the fusion). $Z$-score RNA-seq values for TCGA samples from esophageal

adenocarcinoma (187 samples) and lung squamous cell carcinoma (187 samples) were downloaded from $\mathrm{cBioPortal}^{55}$.

Gene-set enrichment analysis. Data for GSEA and expression of neuroendocrine markers for GSEA analysis, and RNA-seq voom-transformed gene expression measurements ${ }^{56}$ were obtained from ref. ${ }^{10}$. GSEA software was downloaded from the Broad Institute GSEA portal (http://software.broadinstitute.org/gsea/index.jsp) and was applied using default parameters and exploiting signal-to-noise metric for gene ranking. The significance of enrichment was estimated using 1000 gene permutations. Heatmap of neuroendocrine genes in small-cell lung cancer cell lines was generated by the GEDAS software 1.1.6 Beta ${ }^{57}$.

Cancer functional event-drug association analysis. Potential confounding factors in our fusion-drug association analysis were identified by implementing a systematic analysis of associations between cancer functional events published by Iorio et al. ${ }^{8}$ for our panel of cell lines and our set of 409 drugs. The 717 cancer functional events used in the analysis included 281 genes with somatic coding mutations, 424 copy number altered chromosomal segments and methylation status for 12 segments that included any gene altered by point mutations. The analysis was conducted as described by Garnett et al. ${ }^{7}$, although our analysis only considered tissue type and microsatellite instability status as covariates.

We identified 101 large-effect size significant cancer functional event-drug associations across 73 drugs when implementing the same cut-offs as in ref. ${ }^{8}$ (FDR $<25 \%, p$ value $<0.001$, and positive and negative Glass Deltas $>1$ ) reported in Supplementary Data 6.

Gene fusion-drug association analysis. The fusion ANOVA model was constructed as per Garnett et al. ${ }^{7}$, but also includes as covariate any cancer functional event that was involved in a significant large-effect size association with a given 
drug. High-throughput drug sensitivity data were generated by the GDSC project (www.cancerRxgene.org) at the Sanger Institute as previously described ${ }^{8}$. Details of compounds screened and cell line sensitivity data are provided in Supplementary Data 5.

CRISPR screening data analysis. sgRNA raw counts and guide annotations were obtained for 274 cell lines screened as part of Project Achilles by the Broad Institute $^{24}$ (Supplementary Data 10), 14 acute myeloid cell lines screened by Wang et al. ${ }^{6}$ (Supplementary Data 11), and 206 cell lines from Project Score ${ }^{23}$ performed at the Sanger Institute (Supplementary Data 12). CRISPR screening data for EGI-1, ESO51, H3118, SAS, SBC-3, and SNU1411 were generated ad hoc for this study at the Sanger Institute, following the CRISPR screening pipeline used to screen cell lines part of the Project Score. sgRNA target positions were converted from GrCh37 to GrCh38 using the NCBI remapper tool (https://www.ncbi.nlm.nih.gov/ genome/tools/remap).

sgRNA raw counts were converted into log fold changes and corrected for CRISPR biases using CRISPRcleanR ${ }^{58}$ (https://github.com/francescojm/ CRISPRcleanR). Corrected log fold changes were scaled to essentials using the scale-to-essentials R function in the CERES package (https://github.com/ cancerdatasci/ceres/blob/master/R/scale_to_essentials.R).

Positions of sgRNA were mapped onto fusion transcripts using a bespoke $\mathrm{R}$ script that takes into consideration: (1) the fusion transcript breakpoint and (2) the mapping location of the guides. Essentially, sgRNA map onto the $5^{\prime}$ gene if the gene is on the positive DNA strand and the guides maps before the position of the fusion breakpoint, or if the gene is on the negative DNA strand and the sgRNA maps after fusion breakpoint. Guides map onto the $3^{\prime}$ gene if the opposite is true.

In order to calculate an FES, we took the following steps: (1) Z-normalized the scaled and corrected log fold changes across all cell lines screened for a given sgRNA; (2) subtracted the mean $Z$-score of non-mapping from that of the mapping sgRNA for both genes involved in a fusion transcript. Where a gene has no nonmapping sgRNA, the difference was taken from zero; (3) values obtained in step 2 for each gene were averaged to produce the FES.

To assign statistical significance for the FES, we performed 10,000 randomizations of all scaled and corrected log fold changes within cell lines. Randomized FES were then calculated for each fusion transcript, as described in the above paragraph. A $p$ value of statistical significance was assigned to each fusion transcript as the fraction of randomized FES that have a higher FES score than the non-randomized FES. For multiple-hypothesis correction, we calculated a false discovery rate for each $p$ value. Calculation of the FES and the subsequent randomization was performed independently for each data resource, since the sgRNA libraries were different in all cases. Based on an empirical analysis of "gold standard" known functional fusions, we removed any significant hits where the difference between the average scaled fold changes of mapping and non-mapping guides is $<0.45$.

Cell viability assay. Cell lines were seeded at different densities $\left(1-3 \times 10^{3}\right.$ cells per well) in $100 \mu \mathrm{l}$ complete growth medium in 96-well plastic culture plates at day 0 . The following day, serial dilutions of drug were added to the cells in an additional $50 \mu$ of medium. Plates were incubated at $37^{\circ} \mathrm{C}$ in $5 \% \mathrm{CO}_{2}$ for 5 days, after which the cell viability was assessed by measuring ATP content through Cell Titer-Glo Luminescent Cell Viability assay (Promega). Luminescence was measured by Envision Multiplate Reader at day 7. Crystal violet growth assays were performed seeding $30-50 \times 10^{3}$ cells in 6-well plates. After $24 \mathrm{~h}$, the medium was replaced adding drugs as indicated. After 7-10 days of treatments, cells were fixed with a solution of 3\% paraformaldehyde and then stained with $0.05 \%$ crystal violet in distilled water.

PDX database. Gene fusion data for 126 pancreatic adenocarcinoma PDX models were downloaded from HuBase database (https://hubase.crownbio.com/; Crown Bioscience International, Santa Clara, CA, USA).

Interphase and fiber FISH. Metaphase chromosomes were prepared from cell lines listed in Supplementary Data 10 using a standard method. Briefly, colcemid (Thermo Fisher Scientific) was added to a final concentration of $0.1 \mathrm{mg} / \mathrm{ml}$ for $1 \mathrm{~h}$, followed by treatment with hypotonic buffer $(0.4 \% \mathrm{KCl}$ in $10 \mathrm{mM}$ HEPES, pH 7.4) for $10 \mathrm{~min}$ and subsequent fixation using 3:1 (v/v) methanol-acetic acid. Human fosmid and bacterial artificial chromosome (BAC) clones containing the genes of interest (Supplementary Table 1) list of BAC and fosmid clones used in the FISH validation) were provided by the clone archive team of the Wellcome Sanger Institute. Probes were generated by whole-genome amplification with GenomePlex Whole Genome Amplification Kits (Sigma-Aldrich), from purified BAC fosmid DNA as described previously ${ }^{59}$. For interphase and metaphase FISH, probes were labeled directly with Atto488-XX-dUTP, Cy3-XX-dUTP, Texas Red-12-dUTP, and Cy5-XX-dUTP (Jena Bioscience), respectively. Slides pre-treatment included a $10 \mathrm{~min}$ fixation in acetone (Sigma-Aldrich), followed by baking at $65^{\circ} \mathrm{C}$ for $1 \mathrm{~h}$. Metaphase spreads on slides were denatured by immersion in an alkaline denaturation solution $(0.5 \mathrm{M} \mathrm{NaOH}, 1.0 \mathrm{M} \mathrm{NaCl})$ for $7-10$ $\mathrm{min}$, followed by rinsing in $1 \mathrm{M}$ Tris- $\mathrm{HCl}(\mathrm{pH}$ 7.4) solution for $3 \mathrm{~min}, 1 \times \mathrm{PBS}$ for 3 $\mathrm{min}$ and dehydration through a 70,90 , and $100 \%$ ethanol series. The probe mix was denatured at $65^{\circ} \mathrm{C}$ for $10 \mathrm{~min}$ before being applied onto the denatured slides. Hybridization was performed at $37^{\circ} \mathrm{C}$ overnight. The post-hybridization washes included a 5 min stringent wash in $1 \times \mathrm{SSC}$ at $73-75^{\circ} \mathrm{C}$, followed by a 5 min rinse in $2 \times$
SSC containing $0.05 \%$ Tween ${ }^{\circ} 20$ (VWR) and a 2 min rinse in $1 \times$ PBS, both at room temperature. Finally, slides were mounted with SlowFade Gold ${ }^{\oplus}$ mounting solution containing 4',6-diamidino-2-phenylindole (DAPI) (Thermo Fisher Scientific). Indirectly labeled probes were used in fiber FISH with single-molecule DNA fibers. Singlemolecule DNA fibers for fiber-FISH were prepared by molecular combing. The threecolor probe set was labeled with biotin-16-dUTP, DNP-11-dUTP, and digoxenin-11dUTP (Jena Bioscience), respectively, and visualized with Cy3-conjugated, FITCconjugated, and Texas Red-conjugated antibodies, with the exception of posthybridization washes, which consisted of three $5 \mathrm{~min}$ washes in $2 \times \operatorname{SSC}$ at $42^{\circ} \mathrm{C}$, instead of two $20 \mathrm{~min}$ washes in $50 \%$ formamide $/ 50 \% 2 \times$ SSC at room temperature. Slides were examined using AxioImager D1 microscope equipped with appropriate narrow-band pass filters for DAPI, Aqua, FITC, Cy3, Texas Red, and Cy5 fluorescence. Digital images capture and processing were carried out using the SmartCapture software (Digital Scientific UK). Ten randomly selected metaphase cells were karyotyped based on the multiplex FISH and DAPI-banding patterns using the SmartType Karyotyper software (Digital Scientific UK).

\section{Data availability}

All data are available in the main text or the Supplementary information. Supplementary Data 10,11 , and 12 are accessible at the following link https://figshare.com/s/ 169e9fa07450ea7cebce.

Received: 9 August 2018 Accepted: 9 April 2019 Published online: 16 May 2019

\section{References}

1. Kumar-Sinha, C., Kalyana-Sundaram, S. \& Chinnaiyan, A. M. Landscape of gene fusions in epithelial cancers: seq and ye shall find. Genome Med. 7, 129 (2015).

2. Druker, B. J. et al. Efficacy and safety of a specific inhibitor of the BCR-ABL tyrosine kinase in chronic myeloid leukemia. N. Engl. J. Med. 344, 1031-1037 (2001).

3. Kwak, E. L. et al. Anaplastic lymphoma kinase inhibition in non-small-cell lung cancer. N. Engl. J. Med. 363, 1693-1703 (2010).

4. Natrajan, R., Tutt, A. N. J. \& Lord, C. J. Driver oncogenes but not as we know them: targetable fusion genes in breast cancer. Cancer Discov. 8, 272-275 (2018).

5. Mertens, F., Johansson, B., Fioretos, T. \& Mitelman, F. The emerging complexity of gene fusions in cancer. Nat. Rev. Cancer 15, 371-381 (2015).

6. Wang, T. et al. Gene essentiality profiling reveals gene networks and synthetic lethal interactions with oncogenic Ras. Cell 168, 890-903.e15 (2017).

7. Garnett, M. J. et al. Systematic identification of genomic markers of drug sensitivity in cancer cells. Nature 483, 570-575 (2012).

8. Iorio, F. et al. A landscape of pharmacogenomic interactions in cancer. Cell 166, 740-754 (2016).

9. van der Meer, D. et al. Cell model passports-a hub for clinical, genetic and functional datasets of preclinical cancer models. Nucleic Acids Res. 47, D923-D929 (2019).

10. Garcia-Alonso, L. et al. Transcription factor activities enhance markers of drug sensitivity in cancer. Cancer Res. 78, 769-780 (2018).

11. Kumar, S., Vo, A. D., Qin, F. \& Li, H. Comparative assessment of methods for the fusion transcripts detection from RNA-Seq data. Sci. Rep. 6, 21597 (2016).

12. McPherson, A. et al. deFuse: an algorithm for gene fusion discovery in tumor RNA-Seq data. PLoS Comput. Biol. 7, e1001138 (2011).

13. Dobin, A. et al. STAR: ultrafast universal RNA-seq aligner. Bioinformatics 29, 15-21 (2013).

14. Kim, D. \& Salzberg, S. L. TopHat-Fusion: an algorithm for discovery of novel fusion transcripts. Genome Biol. 12, R72 (2011).

15. Gao, Q. et al. Driver fusions and their implications in the development and treatment of human cancers. Cell Rep. 23, 227-238.e3 (2018).

16. Seshagiri, S. et al. Recurrent R-spondin fusions in colon cancer. Nature 488, 660-664 (2012)

17. Davies, K. D. \& Doebele, R. C. Molecular pathways: ROS1 fusion proteins in cancer. Clin. Cancer Res. 19, 4040-4045 (2013).

18. Shaw, A. T. et al. Crizotinib in ROS1-rearranged non-small-cell lung cancer. N. Engl. J. Med. 371, 1963-1971 (2014).

19. Davare, M. A. et al. Foretinib is a potent inhibitor of oncogenic ROS1 fusion proteins. Proc. Natl. Acad. Sci. USA 110, 19519-19524 (2013).

20. Nik-Zainal, S. et al. Landscape of somatic mutations in 560 breast cancer whole-genome sequences. Nature 534, 47-54 (2016).

21. Cokelaer, T. et al. GDSCTools for mining pharmacogenomic interactions in cancer. Bioinformatics 34, 1226-1228 (2018).

22. Chen, Z. et al. Aberrantly activated AREG-EGFR signaling is required for the growth and survival of CRTC1-MAML2 fusion-positive mucoepidermoid carcinoma cells. Oncogene 33, 3869-3877 (2014). 
23. Behan, F. M. et al. Prioritization of cancer therapeutic targets using CRISPRCas9 screens. Nature 568, 511-516 (2019).

24. Meyers, R. M. et al. Computational correction of copy number effect improves specificity of CRISPR-Cas9 essentiality screens in cancer cells. Nat. Genet. 49, 1779-1784 (2017).

25. Aguirre, A. J. et al. Genomic copy number dictates a gene-independent cell response to CRISPR/Cas9 targeting. Cancer Discov. 6, 914-929 (2016).

26. Forbes, S. A. et al. COSMIC: somatic cancer genetics at high-resolution. Nucleic Acids Res. 45, D777-D783 (2017).

27. Jones, D. T. W. et al. Oncogenic RAF1 rearrangement and a novel BRAF mutation as alternatives to KIAA1549:BRAF fusion in activating the MAPK pathway in pilocytic astrocytoma. Oncogene 28, 2119-2123 (2009).

28. Palanisamy, N. et al. Rearrangements of the RAF kinase pathway in prostate cancer, gastric cancer and melanoma. Nat. Med. 16, 793-798 (2010).

29. Chmielecki, J. et al. Comprehensive genomic profiling of pancreatic acinar cell carcinomas identifies recurrent RAF fusions and frequent inactivation of DNA repair genes. Cancer Discov. 4, 1398-1405 (2014).

30. Cancer Genome Atlas Research Network. Integrated genomic characterization of pancreatic ductal adenocarcinoma. Cancer Cell 32, 185-203.e13 (2017).

31. Giacomini, C. P. et al. Breakpoint analysis of transcriptional and genomic profiles uncovers novel gene fusions spanning multiple human cancer types. PLoS Genet. 9, e1003464 (2013).

32. French, C. A. NUT midline carcinoma. Cancer Genet. Cytogenet. 203, 16-20 (2010).

33. Stathis, A. et al. Clinical response of carcinomas harboring the BRD4-NUT oncoprotein to the targeted bromodomain inhibitor OTX015/MK-8628. Cancer Discov. 6, 492-500 (2016).

34. Miyamoto, $\mathrm{H}$. Establishment and characterization of an adriamycin-resistant subline of human small cell lung cancer cells. Acta Med. Okayama 40, 65-73 (1986).

35. French, C. A. et al. NSD3-NUT fusion oncoprotein in NUT midline carcinoma: implications for a novel oncogenic mechanism. Cancer Discov. 4, 928-941 (2014).

36. Sholl, L. M. et al. Primary pulmonary NUT midline carcinoma: clinical, radiographic, and pathologic characterizations. J. Thorac. Oncol. 10, 951-959 (2015).

37. Taniyama, T. K. et al. Clinicopathological features in young patients treated for small-cell lung cancer: significance of immunohistological and molecular analyses. Clin. Lung Cancer 15, 244-247 (2014).

38. Schaefer, I.-M., Dal Cin, P., Fletcher, C. D. M., Hanna, G. J. \& French, C. A. CIC-NUTM1 fusion: a case which expands the spectrum of NUT-rearranged epithelioid malignancies. Genes Chromosomes Cancer. https://doi.org/10.1002/ gcc.3 (2018).

39. Storm, E. E. et al. Targeting PTPRK-RSPO3 colon tumours promotes differentiation and loss of stem-cell function. Nature 529, 97-100 (2016).

40. Russo, M. et al. Reliance upon ancestral mutations is maintained in colorectal cancers that heterogeneously evolve during targeted therapies. Nat. Commun. 9, 2287 (2018).

41. Picco, G. et al. Loss of AXIN1 drives acquired resistance to WNT pathway blockade in colorectal cancer cells carrying RSPO3 fusions. EMBO Mol. Med. 9, 293-303 (2017).

42. Valouev, A. et al. Discovery of recurrent structural variants in nasopharyngeal carcinoma. Genome Res. 24, 300-309 (2014).

43. Lee, K.-W. et al. Significant association of oncogene YAP1 with poor prognosis and cetuximab resistance in colorectal cancer patients. Clin. Cancer Res. 21, 357-364 (2015).

44. Panciera, T., Azzolin, L., Cordenonsi, M. \& Piccolo, S. Mechanobiology of YAP and TAZ in physiology and disease. Nat. Rev. Mol. Cell. Biol. 18, 758-770 (2017).

45. Kitagawa, M. Notch signalling in the nucleus: roles of Mastermind-like (MAML) transcriptional coactivators. J. Biochem. 159, 287-294 (2016).

46. $\mathrm{Hu}, \mathrm{X}$. et al. TumorFusions: an integrative resource for cancer-associated transcript fusions. Nucleic Acids Res. 46, D1144-D1149 (2018).

47. Klijn, C. et al. A comprehensive transcriptional portrait of human cancer cell lines. Nat. Biotechnol. 33, 306-312 (2015).

48. Pietrantonio, F. et al. ALK, ROS1, and NTRK rearrangements in metastatic colorectal cancer. J. Natl. Cancer Inst. 109, https://doi.org/10.1093/jnci/djx089 (2017).

49. Drilon, A. et al. Efficacy of larotrectinib in TRK fusion-positive cancers in adults and children. N. Engl. J. Med. 378, 731-739 (2018).

50. Drilon, A. et al. Safety and antitumor activity of the multitargeted Pan-TRK, ROS1, and ALK inhibitor entrectinib: combined results from two phase I trials (ALKA-372-001 STARTRK-1). Cancer Discov. 7, 400-409 (2017).

51. Prahallad, A. et al. Unresponsiveness of colon cancer to BRAF(V600E) inhibition through feedback activation of EGFR. Nature 483, 100-103 (2012).

52. Nakatani, K. et al. Targeting the Hippo signalling pathway for cancer treatment. J. Biochem. 161, 237-244 (2017).
53. GTEx Consortium. The Genotype-Tissue Expression (GTEx) project. Nat. Genet. 45, 580-585 (2013).

54. Iorio, F. et al. Efficient randomization of biological networks while preserving functional characterization of individual nodes. BMC Bioinforma. 17, 542 (2016).

55. Gao, J. et al. Integrative analysis of complex cancer genomics and clinical profiles using the cBioPortal. Sci. Signal. 6, 11 (2013).

56. Law, C. W., Chen, Y., Shi, W. \& Smyth, G. K. voom: precision weights unlock linear model analysis tools for RNA-seq read counts. Genome Biol. 15, R29 (2014).

57. Fu, L. \& Medico, E. FLAME, a novel fuzzy clustering method for the analysis of DNA microarray data. BMC Bioinforma. 8, 3 (2007).

58. Iorio, F. et al. Unsupervised correction of gene-independent cell responses to CRISPR-Cas9 targeting. BMC Genom. 19, 604 (2018).

59. Gribble, S. M. et al. Massively parallel sequencing reveals the complex structure of an irradiated human chromosome on a mouse background in the Tc1 model of Down syndrome. PLoS ONE 8, e60482 (2013).

\section{Acknowledgements}

We thank the Garnett laboratory, Cellular Genetics and Phenotyping facility, and drug screening teams at the Sanger Institute for data generation and assistance. We also thank Sandra Louzada for support with DNA probe preparation. We would also like to thank Serena Nik-Zainal and Andrea Degasperi for useful discussions.

\section{Author contributions}

The project was conceived by M.J.G., G.P., E.D.C., and U.M. Molecular biology experiments were designed by G.P. and E.D.C., and performed by G.P. Computational analyses were designed by E.D.C. and G.P. and performed by E.D.C. RNA-seq data pre-processing was performed by G.B. and A.M. Drug screening data generation was led by M.J.G., C.H.B., and U.M. Gene expression analysis was performed by L.G.A. GSEA was performed by G.P. CRISPR-Cas9 screening data was provided by F.M.B., F.I., E.G., E.S., K.Y. and M.J.G. Statistical and computational advice was provided by E.G. and F.I. PCR validations was performed by E.A. FISH experiments were performed by B.F., R.B., and F.Y. Project supervision was assisted by A.B., D.D., J.S-R., and E.S. The manuscript was written by M.J.G., G.P., and E.D.C. All authors edited and approved the manuscript. Funding was acquired by M.J.G. and J.S-R. The project was administered by M.J.G.

\section{Additional information}

Supplementary Information accompanies this paper at https://doi.org/10.1038/s41467019-09940-1.

Competing interests: E.S. and D.D are employees of GSK. U.M. is an employee of AstraZeneca. M.J.G receives funding from AstraZeneca and performed consultancy for Sanofi. This works was funded by Open Targets which is a public-private initiative involving industry and academia. The remaining authors declare no competing interests

Reprints and permission information is available online at http://npg.nature.com/ reprintsandpermissions/

Journal peer review information: Nature Communications thanks Charles Mullighan and the other, anonymous, reviewer(s) for their contribution to the peer review of this work. Peer reviewer reports are available.

Publisher's note: Springer Nature remains neutral with regard to jurisdictional claims in published maps and institutional affiliations.

Open Access This article is licensed under a Creative Commons Attribution 4.0 International License, which permits use, sharing, adaptation, distribution and reproduction in any medium or format, as long as you give appropriate credit to the original author(s) and the source, provide a link to the Creative Commons license, and indicate if changes were made. The images or other third party material in this article are included in the article's Creative Commons license, unless indicated otherwise in a credit line to the material. If material is not included in the article's Creative Commons license and your intended use is not permitted by statutory regulation or exceeds the permitted use, you will need to obtain permission directly from the copyright holder. To view a copy of this license, visit http://creativecommons.org/ licenses/by/4.0/

(C) The Author(s) 2019 